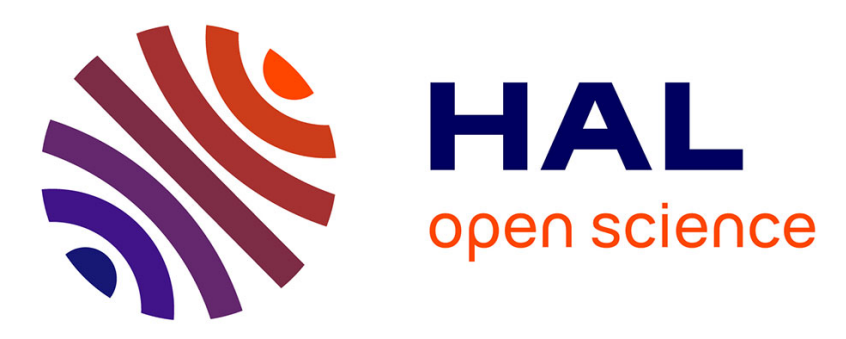

\title{
River Corridor Model Constrained by Time-Lapse Seismic Acquisition
}

Marine Dangeard, Agnès Rivière, Ludovic Bodet, S. Schneider, R. Guérin, Damien Jougnot, Alexis Maineult

\section{- To cite this version:}

Marine Dangeard, Agnès Rivière, Ludovic Bodet, S. Schneider, R. Guérin, et al.. River Corridor Model Constrained by Time-Lapse Seismic Acquisition. Water Resources Research, 2021, 57 (10), pp.e2020WR028911. 10.1029/2020WR028911 . hal-03404796

\section{HAL Id: hal-03404796 https://hal.science/hal-03404796}

Submitted on 26 Oct 2021

HAL is a multi-disciplinary open access archive for the deposit and dissemination of scientific research documents, whether they are published or not. The documents may come from teaching and research institutions in France or abroad, or from public or private research centers.
L'archive ouverte pluridisciplinaire HAL, est destinée au dépôt et à la diffusion de documents scientifiques de niveau recherche, publiés ou non, émanant des établissements d'enseignement et de recherche français ou étrangers, des laboratoires publics ou privés. 


\section{Water Resources Research}

\section{RESEARCH ARTICLE \\ 10.1029/2020WR028911 \\ Key Points: \\ - Mapping hydrofacies, expanding the modeling domain and building stronger model constraints in space and time by using a multimethod approach \\ - Capturing the local spatial and temporal dynamic of the water table (WT) with high resolution time- lapse seismic acquisitions \\ - Using the WT geometry dynamics to calibrate and perform fully integrated $2 \mathrm{D}$ river corridor modeling}

Correspondence to:

A. Rivière,

agnes.riviere@mines-paristech.fr

Citation:

Dangeard, M., Rivière, A., Bodet, L., Schneider, S., Guérin, R., Jougnot, D. $\&$ Maineult, A. (2021). River corridor model constrained by time-lapse seismic acquisition. Water Resources Research, 57, e2020WR028911. https:// doi.org/10.1029/2020WR028911

Received 30 SEP 2020 Accepted 24 SEP 2021
(C) 2021. American Geophysical Union. All Rights Reserved.

\section{River Corridor Model Constrained by Time-Lapse Seismic Acquisition}

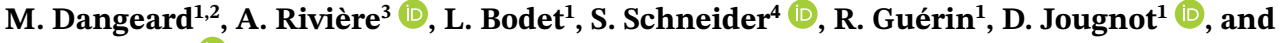 \\ A. Maineult ${ }^{1}$ iD \\ ${ }^{1}$ Sorbonne Université, CNRS, EPHE, Paris, France, ${ }^{2}$ Now at SNCF Réseau, Paris, France, ${ }^{3}$ Geosciences Department, \\ Mines ParisTech, PSL University, Paris, France, ${ }^{4}$ SUEZ RR IWS REMEDIATION, Lyon, France
}

\begin{abstract}
A new hydrogeophysical approach was developed to reveal the spatial and temporal dynamic flow pathways inside a river corridor as well as the pattern of transient surface water and the groundwater (SW-GW) exchanges. Obtaining such information from traditional hydrological data remains a challenge, both in terms of accuracy and validation. As there is no direct measurement of these fluxes, estimating dynamic pathways and SW-GW exchanges is frequently tackled with the help of numerical models. Yet, the definition of initial and boundary conditions is generally based on poorly constrained assumptions and restrained to the location of piezometers, due to the lack of knowledge of the water table (WT) geometry. We provide a methodology to build stronger constraints to the numerical simulation and the hydrodynamic parameters calibration, both in space and time, by using a multi-method approach. On the Orgeval Critical Zone observatory (France), we show how a thorough interpretation of highresolution geophysical images, combined with geotechnical data, helps to resolve subsurface structural heterogeneity. This coupling provides a detailed distribution of hydrofacies, valuable prior information about the associated hydrodynamic properties distribution. The temporal dynamic of the WT table can be locally captured with high-resolution time-lapse seismic acquisitions. Time-lapse variations are interpreted as temporal changes in the saturated-unsaturated zone continuum. Each seismic snapshot is then thoroughly inverted to image spatial WT variations. This posterior geophysical information is then used to calibrate the hydrogeological modeling domain. The use of the WT improves the estimation of transient stream-aquifer exchanges, especially during the rain event.
\end{abstract}

\section{Introduction}

The critical zone $(\mathrm{CZ})$ is characterized by its interface nature between human activities, life-sustaining ecosystems and physical objects. The National Research Council (NRC, 2001) defined it as the place "where water, rock, air, and life meet in a dynamic interplay" (e.g., Chorover et al., 2007). The interfaces between those different hydrosystems sub-compartments are the place where important physical and biogeochemical processes can take place through the mixing of water with different temperatures or chemistry and biogeochemical reactions (Boano et al., 2010; Brunner et al., 2017; Fan et al., 2019; Shuai et al., 2017; Trauth \& Fleckenstein, 2017; Trauth et al., 2018). These reactive transport processes yield different ecosystem services (Griebler \& Avramov, 2015) such as the provision of clean drinking water, agricultural uses, or the attenuation of pollutants (Conant et al., 2019, Harvey \& Gooseff, 2015; Kalbus et al., 2006; Krause et al., 2011; Ranalli \& Macalady, 2010; Trauth et al., 2018; Vidon and Hill, 2004, 2006; Zarnetske et al., 2011, 2012).

Such ecosystemic services are often to be addressed along the river corridors including river channels, riparian zones, fluvial deposits, alluvial and flood plains. River corridors are thus complex zones where surface water (SW) and groundwater (GW) interactions are particularly critical (Krause, Boano, et al., 2014; Krause, Freer, et al., 2014; Winter et al., 1998; Woessner, 2000). Yet, the river dynamics and history induce strong geological heterogeneities and complex spatio-temporal preferential pathways due to the aquifer parameters, such as hydraulic conductivities or porosities (Cardenas, 2009; Cardenas et al., 2004; Pinay et al., 2015; Pryshlak et al., 2015; Sawyer \& Cardenas, 2009; Song et al., 2018). These subsurface heterogeneities exert an important control on the water table (WT) geometry. Preferential pathways within the river corridor modify nutrient cycling and other biogeochemical processes (such as the dispersion), which impact chemical budgets (e.g., Bernard-Jannin et al., 2017; Wildenschild et al., 1994) and biological activity. The reactive potential along a flow path depends on the time that a water parcel stays in contact with the reactive media (Oldham 
et al., 2013). This makes it essential to model water fluxes and the flow path within river corridors. There is however no direct measurement of the SW-GW fluxes and no method to characterize flow paths, at least in a straightforward manner. Despite these difficulties, the dynamics of water fluxes between streams and aquifers is characterized thanks to the three last decades of research, including: the response of SW-GW exchanges to varying streamflow (Dudley-Southern \& Binley, 2015), the impact of the streambed heterogeneities (Irvine et al., 2012; Tang et al., 2015), as well as the effect of clogging layer (Brunner et al., 2011; Rivière et al., 2014).

In addition, close to the river, the Dupuit-Forchheimer assumption (i.e., vertical flow is negligible under dominant horizontal flows over vertical flows) is not valid in the aquifer near a stream. The direction of GW flow close to the river may have a vertical component, thereby violating the Dupuit-Forchheimer assumption of zero resistance to vertical flow. Morel-Seytoux (2009) presents a promising approach to estimate the distance between the lateral boundary condition and the river where the Dupuit-Forchheimer approximation should be considered legitimate. However, without a piezometric level (PL) available at this location, the levels recorded in the banks are commonly used as a Dirichlet condition in the edge of the model (e.g., Mouhri et al., 2013).

Estimating the spatial variations of aquifer parameters is one of the most difficult tasks to ensure a correct calibration of hydrogeological models and, therefore, a correct description of flow and transport into a river corridor. Numerous studies have modeled the impact of heterogeneities of subsurface on hyporheic exchange fluxes, residence times (Sawyer \& Cardenas, 2009), flow patterns, and reactive solute spatial distribution (Bardini et al., 2013).

Impacts of heterogeneous streambed sediments have been described for stratified sediments by Marion et al. (2008) and Packman et al. (2006) and discussed for different scales in Conant (2004), Fox et al. (2016), Genereux et al. (2008), Hester et al. (2019), Kennedy et al. (2009a, 2009b), Krause et al. (2012), Krause et al. (2013), Leek et al. (2009), Rosenberry and Pitlick (2009), Salehin et al. (2004), and Weatherill et al. (2014). In addition, previous modeling studies have attempted to quantify the scale dependent relevance of low-conductivity zones in streambed sediments (Bardini et al., 2013; Gomez-Velez et al., 2014; Laube et al., 2018; Sawyer, 2015; Sawyer \& Cardenas, 2009). These studies mainly represented riverbed heterogeneity using geostatistical approaches, which rely on generating spatial variability in conductivity or simple geometry. The main issue of these previous studies is to validate the simulated flow pathways from field measurements. The importance of the spatial variability of aquifers properties (aquifer geometry and hydrofacies) and associated consequences on WT, flow, and transport processes have been widely recognized and studied (Cardenas et al., 2004; de Marsily et al., 2005; Freeze \& Witherspoon, 1967; Irvine et al., 2012; Kalbus et al., 2009). Fixing the WT determines the flow distribution at any depth and the overlying recharge (Goderniaux et al., 2013; Liang et al., 2012; Sanford, 2002). By consequence, calibrating the WT geometry is the new approach to identify the flow pathways. In general, the geometry of the WT is interpolated when several piezometers are available but never estimated directly by a field measurement.

Recent developments in multi-scale geophysical imaging and hydrogeophysics provide a great toolbox to better characterize heterogeneities along streams (Binley et al., 2010, 2015; McLachlan et al., 2017; Parsekian et al., 2015) but no methods provide the WT geometry dynamics that allow to calibrate the flow path way. Geophysical techniques sensitive to water fluxes such as self-potential methods for instance, have been naturally deployed in various contexts to study SW-GW exchanges (e.g., Linde et al., 2011; Valois et al., 2018). The combination of approaches is the key to understanding the complex dynamic of SW-GW interaction and the WT movement (Kalbus et al., 2006; Linde et al., 2010; Schneider et al., 2011). For example, studies suggest combinations with temperature monitoring, electrical resistivity tomography (ERT; Coscia et al., 2011; Singha et al., 2008), ground-penetrating radar, boreholes techniques (Doetsch et al., 2012). The importance of geophysical monitoring (e.g., continuous measurements) and/or the repetition of acquisitions in time (e.g., time-lapse measurements) are crucial to go from structural and static property imaging to dynamic process-based imaging approaches (Binley et al., 2015; Blazevic et al., 2020; Dangeard et al., 2018; Descloîtres et al., 2003; Uhlemann et al., 2017; Watlet et al., 2018).

Time-lapse seismic methods have been developed to track temporal changes of Earth's near-surface properties, for instance, linked to weathering effects (Bergamo et al., 2016a; Bergamo \& Socco, 2016; Ikeda 
et al., 2017; Lu, 2014), water level variations (Lu \& Likos, 2004; Pasquet, Bodet, et al., 2016; Pasquet, Bodet, Longuevergne, et al., 2015), permafrost thaw (Ajo-Franklin et al., 2017) or deep fluid storage (Arts et al., 2004; Chadwick et al., 2010). Seismic techniques recently became part of the hydrogeophysics' toolbox to study not only the CZ architecture (St. Clair et al., 2015) but also its water content (Cameron \& Knapp, 2009; Grelle \& Guadagno, 2009; Konstantaki et al., 2013; Pasquet, Bodet, Dhemaied, et al., 2015; Pasquet, Bodet, Longuevergne, et al., 2015; Pasquet, Holbrook, et al., 2016; Pride, 2005). Acquisition setups usually consist in a collection of geophones implanted along linear profiles or arrays, at the surface of the ground excited by a portable mechanical source. Depending on the dimensions of the setups, the density of the arrays and the energy of the sources, the recorded wavefields can be analyzed to image contrasts in mechanical properties of the near-surface (down to 1-100 m depths of investigation (DOI) and with 0.1$10 \mathrm{~m}$ scale resolutions) using the standard equipment available for environmental applications (Adelinet et al., 2018; Mari \& Porel, 2008; Uhlemann et al., 2016).

The most popular interpretation method is refraction tomography which consists of picking first arrivals times of the wavefield that are then inverted for pressure $(\mathrm{P})$ or shear $(\mathrm{S})$ waves velocity models (VP or VS, depending on the type of sources and sensors). As an alternative to $S$ wave refraction tomography, the recorded wavefield can also be processed to extract surface-wave dispersion data that are then inverted for VS models (e.g., Pasquet \& Bodet, 2017). By definition, VP and VS are showing partially decoupled behaviors in the presence of fluids (Biot, 1956a, 1956b). VP/VS or Poisson's ratio ( $v$ ) are usually estimated to image it in materials. These parameters recently proved efficient in the description of spatial and temporal variations of water content in the CZ, along the continuum between the saturated and unsaturated zones (e.g., Pasquet, Bodet, Dhemaied, et al., 2015; Pasquet, Bodet, Longuevergne, et al., 2015). A time-lapse implementation of this approach has been developed by Dangeard et al. (2018) associated with a processing workflow to thoroughly estimate $P$ wave first arrivals and surface-wave dispersion picking errors. Dangeard et al. (2018) showed it is possible to properly consider the WT carried by seismic data, at least in a qualitative manner. Unlike conventional techniques like ERT measurements, seismic methods can be used to discriminate the interface between dry and fully saturated zones, especially in conductive soils.

We develop a new coupled hydrogeophysical approach integrating the ERT, time-lapse seismic, the piezometric data and hydrogeological modeling that provide a significant contribution to quantify the flow pathway and SW-GW exchanges by the use of estimated WT geometries. This study is applied to the outlet of the Orgeval CZ observatory (France). Our methodology is transferable to other environments where it is possible to implant geophones. We targeted a $55 \mathrm{~m}$ long section intersecting the river equipped with a high-frequency monitoring station (Mouhri et al., 2013). We performed high-resolution ERT, dynamic penetrometer tests, and Auger soundings to define and map the heterogeneities of the hydrofacies field to be used in a hydrodynamic model. Time-lapse seismic acquisitions with a 2 month time step over 6 months were performed to qualitatively observe both spatial and temporal water content changes. With the help of strong a priori information about the medium structure, time-lapse data were inverted to delineate the WT geometry along the cross-section, outside the usual limits imposed by the piezometers. It provided updated constraints as well as initial and boundary conditions to the hydrodynamic model by extrapolating point continuous data from piezometers. A grid search to optimize the hydrodynamic parameters of each hydrofacies was performed to reproduce the WT and the PL time series. From this analysis, the functioning of the river corridor is described with a special attention during the flood event.

\section{The Orgeval Critical Zone Observatory (CZO)}

The studied site is located on the Orgeval CZO (Gaillardet et al., 2018), $70 \mathrm{~km}$ east from Paris in Seine-etMarne (France). It is a headwater representative of the Seine watershed, characterized by a large agricultural cover (80\% of the area; Loumagne \& Tallec., 2013). It is facing considerable issues related to anthropic impacts on streamflow and water quality, with a particular focus on agricultural activities (Floury et al., 2018; Garnier et al., 2016; Mariotti \& Letolle, 1977) and risks related to extreme events (floods, droughts; Neira et al., 2020). As a consequence, Orgeval CZO has been monitored since 1960 (Hlavek \& Dubreuil, 1965; Loumagne \& Tallec., 2013). We focus on the Avenelles stream which drains a $46 \mathrm{~km}^{2}$ sub-catchment (Figure 1a). The hydrogeological functioning is influenced by a multi-layered aquifer system composed of two main horizontal geological units: the Oligocene and the Eocene aquifers, separated by a clayey aquitard 
a.

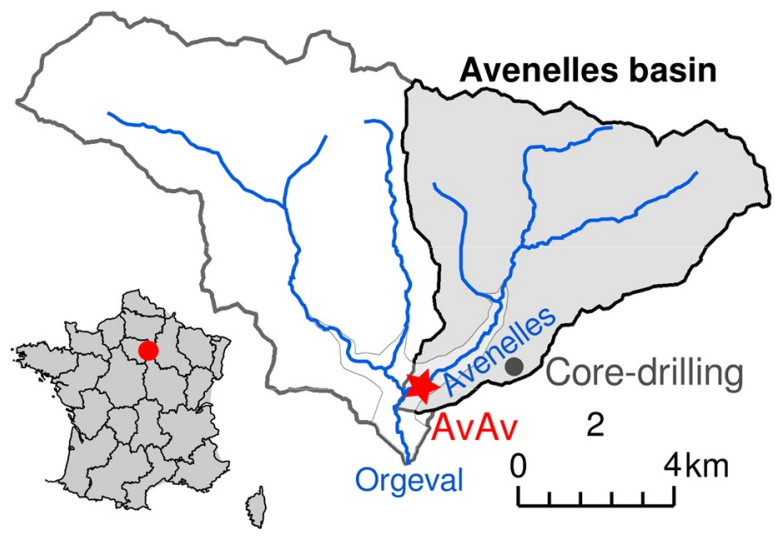

b.

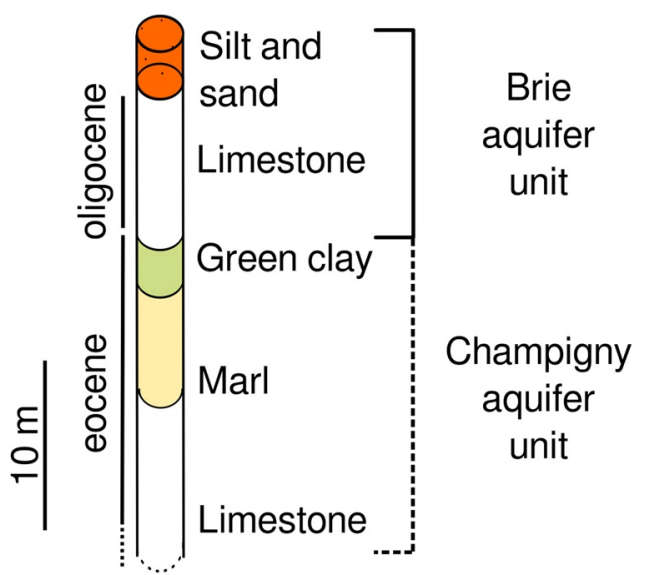

c.

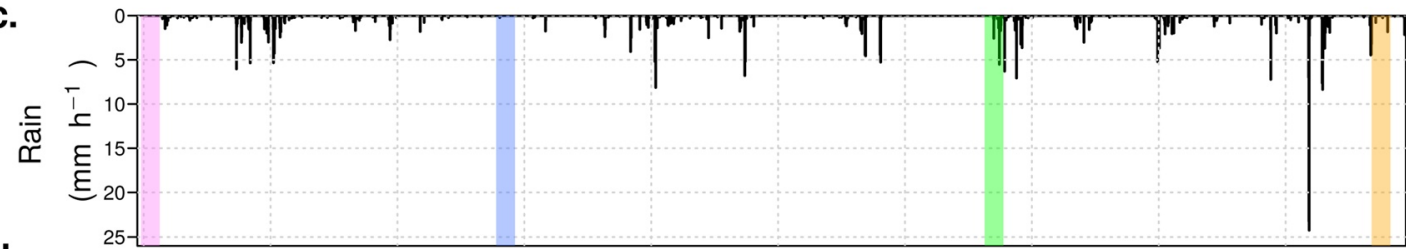

d.

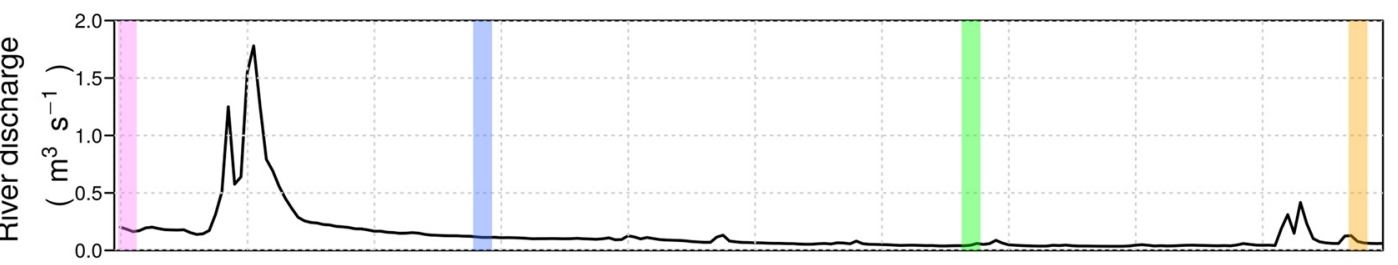

e.

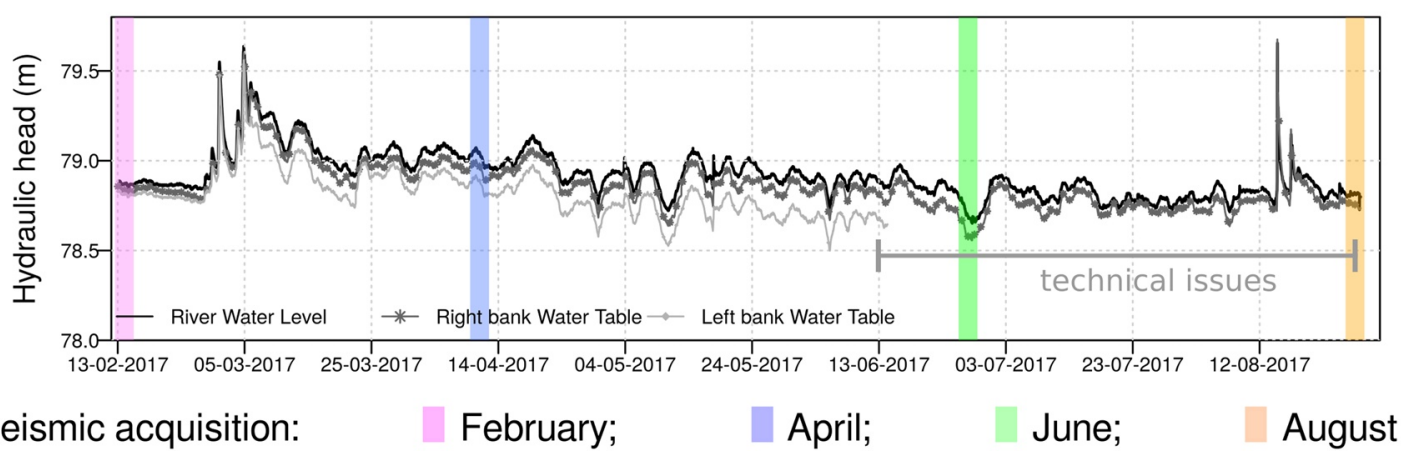

Figure 1. (a) Location of the Avenelles catchment and the studied hotspot (AvAv, red star). (b) Geological log of the area, from the core-drilling at the black spot on the map. (c) Rainfalls recorded at the Boissy-le-Châtel meteorological station between February 14th and August 28 th, 2017 (dates of the first and last seismic acquisitions) (d) River discharge recorded 200 m upstream of the station AvAv (e) PL recorded during the same period at AvAv in the river (black), and in right (dark gray) and left (light gray) bank piezometers. During June, the sensor of the left bank stopped functioning (see Figure 2).

(green clays and marls, Figure 1b; Mouhri et al., 2013). Mouhri et al. (2013) described the geometry of sediment layers at the basin scale thanks to several geological and geophysical studies and core samples. The surface of the basin is covered with quaternary loam deposits (loamy silt with high porosity sand lenses). The Oligocene partly silicified lacustrine limestone formation constitutes an unconfined shallow aquifer (the Brie aquifer). This aquifer constitutes the main water resource of the basin (Gallois et al., 2019). The upper part of the Eocene aquifer is a mixture of white marls, limestones, and evaporites formed in a coastal plain environment (Mégnien, 1973). Below, the Middle Eocene limestone hosts the unconfined Champigny aquifer containing gypsum lenses (Mégnien, 1973) deployed high-frequency monitoring of hydrogeological parameters (temperature, PL) thanks to LOcal MOnitoring Station (LOMOS). 


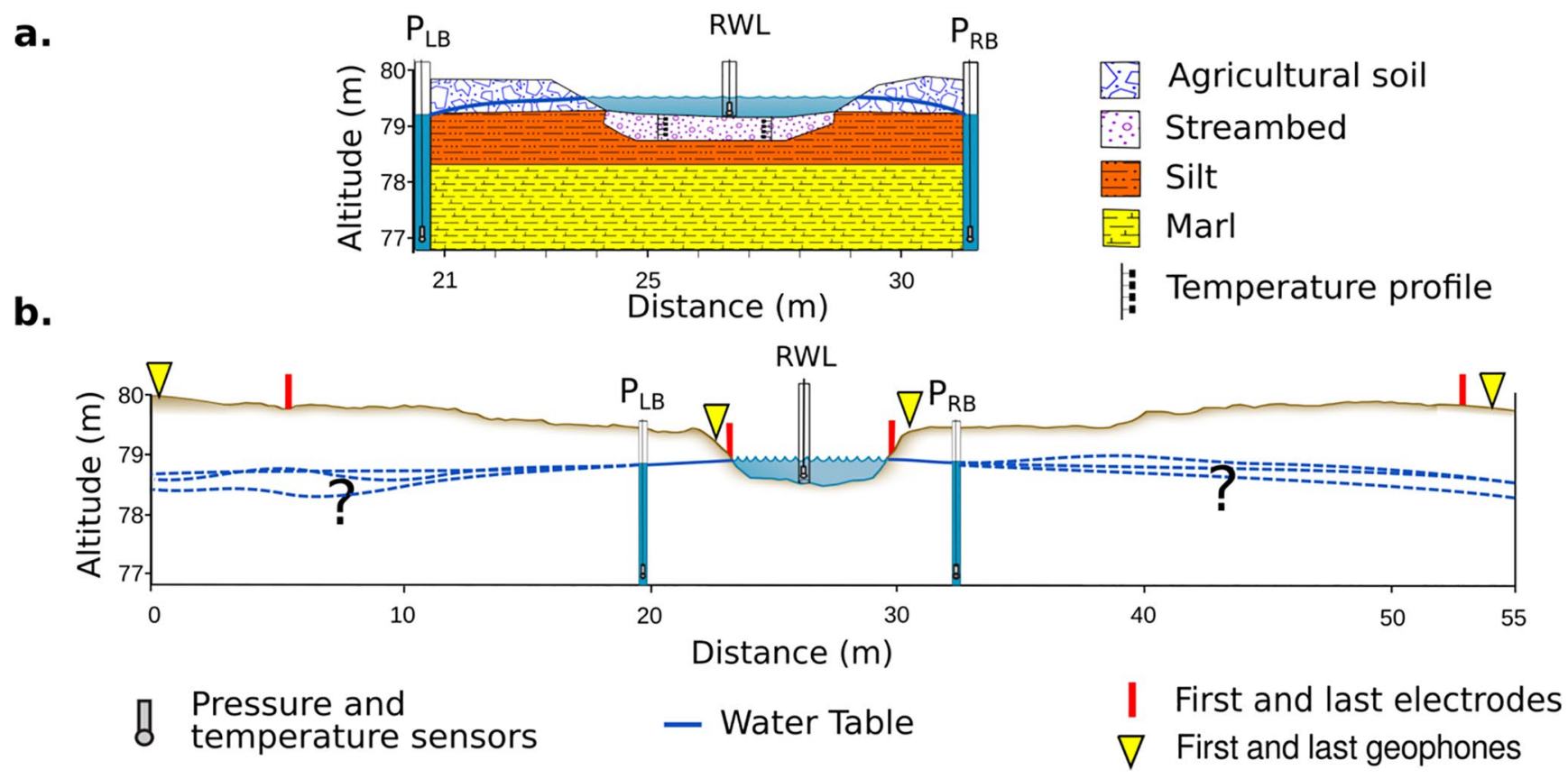

Figure 2. (a) Simple confined between the PL in the Left Bank $\left(\mathrm{P}_{\mathrm{LB}}\right)$ and the Right Bank $\left(\mathrm{P}_{\mathrm{RB}}\right)$ at AvAv LOMOS (recording the RWL). The temperature is measured in streambed as well. (b) Lateral extent and topography along the geophysical section deployed to expand the modeling domain, image hydrofacies with ERT (first and last electrodes displayed as red bars), map the WT (dashed blue line) with time-lapse seismic (first and last geophones displayed as yellow triangles) and the question mark mentions to the geometry of the water table.

The research undertaken here is concentrated at the station "AvAv" located at the outlet of the basin (Figure 1a). By its position in the basin, this station is of great interest for understanding the biogeochemical cycle of the basin (Floury et al., 2018; Mouchel et al., 2019). This station is in the floodplain of the Avenelles river. The floodplain is a few-meters thick, heterogeneous layer consisting of various deposits; clays, silts, sands, and gravels. Figures 1c-1e respectively show rainfall, river discharge, and PL variations recorded between February 2017 and August 2017 at a 15 min interval. Unfortunately, the pressure sensor in the left bank stopped functioning in June. April was the driest month. The major flood events took place from 24 February to 5 March and from 13-17 August, with a maximum stream discharge of $1.78 \mathrm{~m}^{3} \mathrm{~s}^{-1}$ on March 5 and $0.42 \mathrm{~m}^{3} \mathrm{~s}^{-1}$ on August 17 (Figure 1d). Summer was particularly wet with a lot of storm events. During the data collection period, RWL was never lower than PL. Therefore, the section 'AvAv' is identified as a downwelling station i.e., the river discharges in the aquifer during the most of the year. Time series of PL and RWL indicate a strong synchronicity of the GW and river systems (Figure 1e). The two banks show similar dynamics during the rising limb of the rain event (before the peak flow), but during the recession, the left one decreases faster. These differences are linked to the heterogeneities of the aquifer. Simultaneous increases of both RWL and WT were linked to the heavy rain events until they reached almost the same level. This is due to the position of the piezometers inside the floodplain.

Figure 2a presents the geological section interpreted by Mouhri et al. (2013) at the basin scale, as a stack of horizontal and homogeneous layers, bounded by the piezometers. Berrhouma (2018) optimized the hydrodynamic parameters set by using the heat as a tracer to describe the hyporheic water fluxes. The PL time series measured in each piezometer were applied as lateral boundary conditions of the model and the recorded RWL is prescribed in the river. The bottom of the cross-section had no boundary condition. According to Berrhouma (2018) simulations, the river loses water into the aquifer for the major part of the year. The objective function was to minimize the differences between the two observed and simulated vertical temperature profiles in the streambed. Therefore, this model accurately characterized the physical processes within the hyporheic zone but not into the riparian zone. The different behavior between the left and right banks were stimulated artificially by imposing hydraulic heads recorded in the piezometers. However, Mouchel et al. (2019) showed that the concentrations of sulfate and magnesium differ between the right and the left banks, which seems linked to the heterogeneities (the concentration of sulfate is higher in 
a.

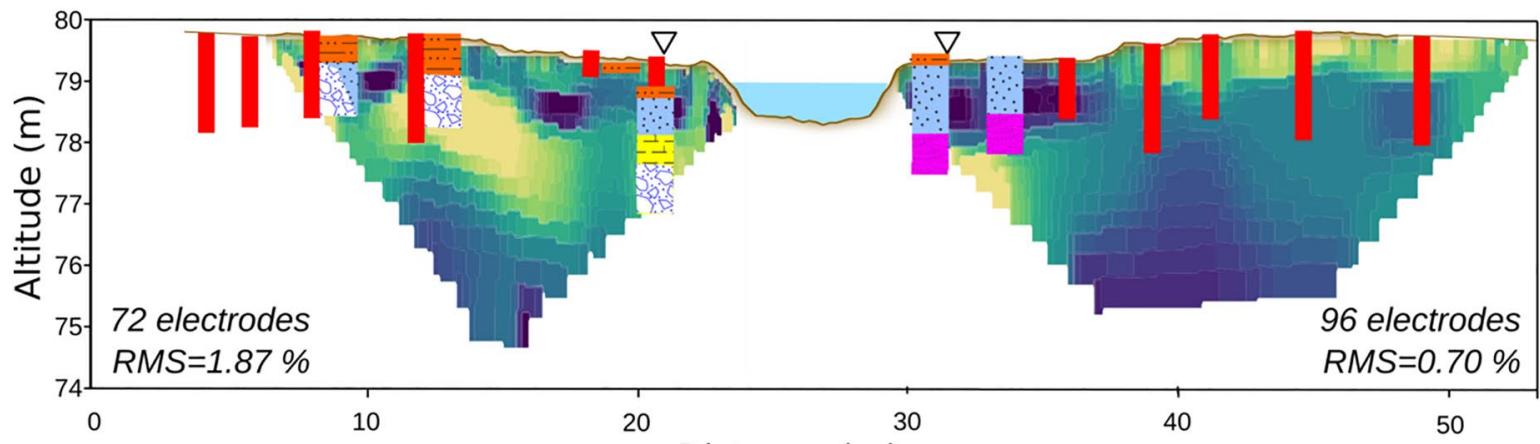

b.

Distance $(m)$
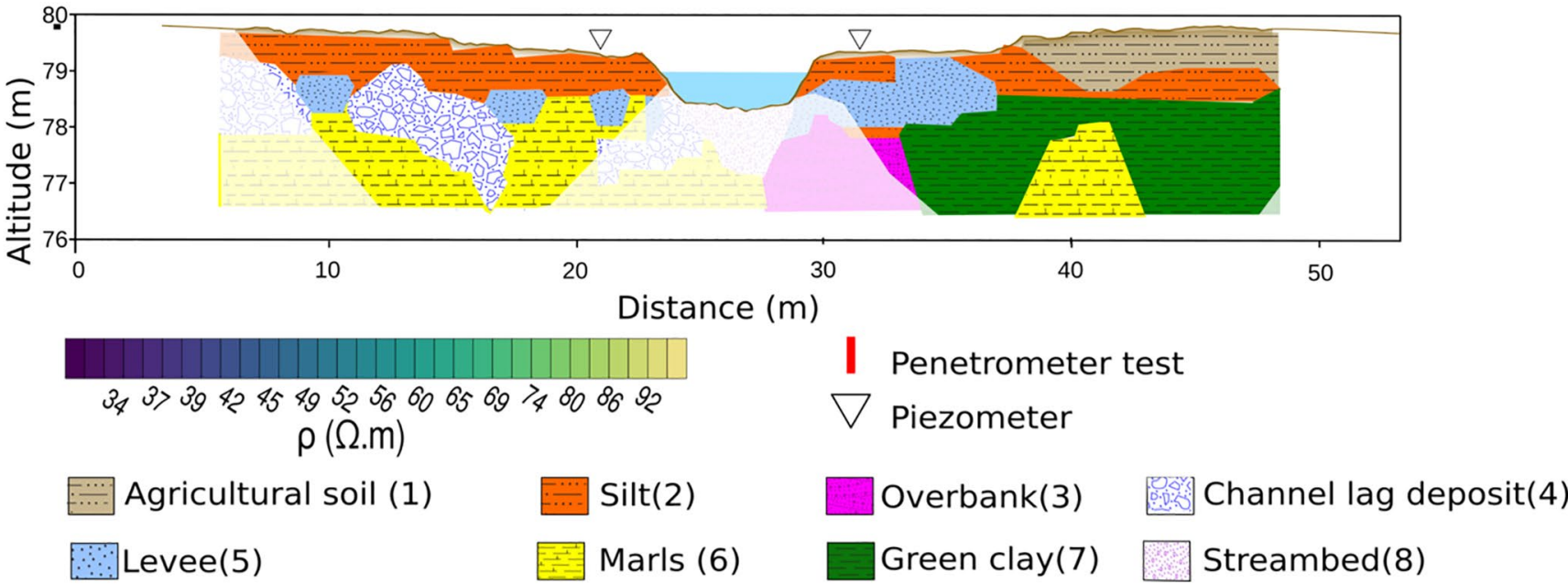

Figure 3. Geotechnical soundings and ERT profiles performed along the ESE-WNW line perpendicular to the Avenelles stream (see Figure 2b). (a) Interpreted resistivities and final Root Mean Square errors given after four iterations of the inversion process described in Loke and Barker (1996). The red lines present the location of penetrometer tests (data sets are presented in Appendix A1). The results of Auger sampling are presented by the logs. (b) Mapping of interpreted lithofacies from correlations between resistivity and geotechnical results. The white shaded areas indicate interpolations and extrapolations of ERT interpretations correlated with available priordata. (1) agricultural soil, (2) the silt, (3) (4) and (5) a succession of flood deposits, (6) the marls and (7) the green clay.

the right bank). In order to properly simulate the stream-aquifer behavior of both banks (at the local scale), these heterogeneities, unfortunately not considered in the simple model of Figure 2a, must be characterized and related to hydraulic properties.

\section{Geotechnics and ERT to Map the Hydrofacies and Expand the Modeling Domain}

In order to describe the hydrofacies distribution at the studied hotspot, we relied on the high-resolution imaging of electrical resistivity which is one of the most discriminating parameters in such a clayey-loamy environment. An ERT profile, perpendicular to the Avenelles stream, was thus deployed in February 2017. A multi-channel resistivimeter with 72-electrode (left bank) and 96-electrode (right bank) Wenner-Schlumberger arrays were used. The electrodes were spaced $0.25 \mathrm{~m}$ apart (as detailed in Appendix A1) in order to obtain a resolution adequate to the heterogeneities expected thanks to our knowledge of the field. 2D resistivity sections were inferred from measured data using the inversion process described in Loke and Barker (1996) (as detailed in Appendix A1). Inversion results for both banks are presented as obtained after four iterations (Figure 3a).

Inverted resistivity ranges were interpreted using: (a) geotechnics, e.g., 13 dynamic penetrometer tests, see data sets in Appendices A2 and A4 Auger soundings, about $1 \mathrm{~m}$ deep (Figure 3a); (b) geological information 
(from the core-drilling); (c) geophysics (ERT images by Mouhri et al., 2013); and (d) prior information about the site (empirical knowledge from experiments and instruments installations performed since the last $10 \mathrm{yr}$ (see Berrhouma, 2018). Under the river, the hydrofacieses were interpolated taking several data sets and sources of information into account: (a) the cores (Figure 2), sampled on both banks during the installation of the piezometers, show variations in facies at depth, close to the river; (b) temperature probes (Berrhouma, 2018; Mouhri, et al., 2013; Rivière, Flipo, et al., 2019; Rivière, Jost et al., 2019; Figure 2) and the mini LOMOS sensor (Cucchi et al., 2018, 2019) installed in the riverbed indicate that the streambed is more than $60 \mathrm{~cm}$ thick; (c) the geometry of the meander itself implies an asymmetry of the streambed layer with a greater thickness on the right bank side. All this information was combined to define the succession of lithological units (Figure 3b). The cross-section (Figure 3b) is actually marked by an asymmetry between the two banks with rather contrasted facieses:

1. (1) agricultural soil about $50 \mathrm{~cm}$ thick identified on the right bank (90 $\Omega . \mathrm{m})$.

2. (2) the silt at the surface (50-60 $\Omega . \mathrm{m})$.

3. (3) (4) and (5) a succession of flood deposits ( $<40$ and $>80 \Omega . m$ ).

4. (6) the marls ( $40 \Omega . \mathrm{m})$ on the left bank.

5. (7) the green clay $(<40 \Omega . \mathrm{m})$ on the right bank.

The heterogeneities on the left bank are related to frequent flood deposits inside the meander characterized by a succession of coarse deposits (channel lag deposit) and fine-grained deposits (levee). On the contrary, the lithology of the right bank is homogeneous and mainly clayey. The streambed of the river was characterized by Berrhouma (2018) as colluvium deposits.

These structures and their important variations along the cross-section confirm the dynamic of the hydrological data detailed earlier (Figure 2a). It is important to note that these lithofacies will be used to determine the parameterization of the hydrofacies. Therefore, the discontinuous geometry of the lithofacies will naturally influence the simulation results (abrupt changes, discontinuities, etc). As two piezometers, located close to the stream inside the floodplain, are not sufficient to describe the WT spatial variations in time in such a heterogeneous medium, 2D time-lapse imaging is required. Time-lapse ERT can be a powerful tool to image saturation changes (Blazevic et al., 2020). Yet, in the clayey contexts of the present study, and with such small water content variations between February and August (Figure 1), the material resistivity does not present variations strong enough to be detected with the setups we used. Pasquet, Bodet, Dhemaied, et al. (2015) for instance performed diachronic ERT experiments at high water and low water periods, in the same area. This study clearly demonstrated that, in these particular materials, the changes in WT depth was only detected by the combined use of $P$ and $S$ wave models (through the computation of Poisson's ratio).

\section{Time-Lapse Seismic to Capture the WT Dynamics and Constrain the Model}

Seismic lines were deployed along the profile from February to August 2017 with a 2 month step. For each acquisition, the equipment, the geometry and acquisition parameters remained similar. In order to ensure optimal positioning of the line over time, a permanent marking system has been used. A $23.75 \mathrm{~m}$ long profile was deployed on each bank (Figure 2b), using a 96-channel seismic recorder with $14 \mathrm{~Hz}$ vertical component geophones $0.25 \mathrm{~m}$ spaced and a $1.25 \mathrm{~kg}$ hammer as source. As detailed in Appendix A and Dangeard (2019), the seismic data set consists of a total number of 97 seismograms per bank at each time step. In June, a storm occurred between the acquisition of the two banks; the acquisition of the left bank was carried out in dry conditions while the acquisition of the right bank was realized in wet conditions. Moreover, due to this storm event, the last seismic shots of the acquisition on the right bank have been perturbed (noise due to the drops). Some seismograms were corrupted and were not fully exploited at the very end of the profile.

Before any time-lapse interpretation, data picking errors must be quantified following the workflow and recommendations by Dangeard et al. (2018). Such analysis makes it possible to differentiate time-lapse variations observed in seismic data due to temporal changes in the vadose zone thickness continuum on one side, from noise or measurement errors on the other side (see details in Appendix A). In this sense, $P$ wave traveltimes were manually picked several times to estimate picking error. Arrival time differences were then calculated between two time steps and picking error was then retrieved. Changes in seismic data that were above the threshold is considered as due to variations in water content. It was then possible to invert VP by 


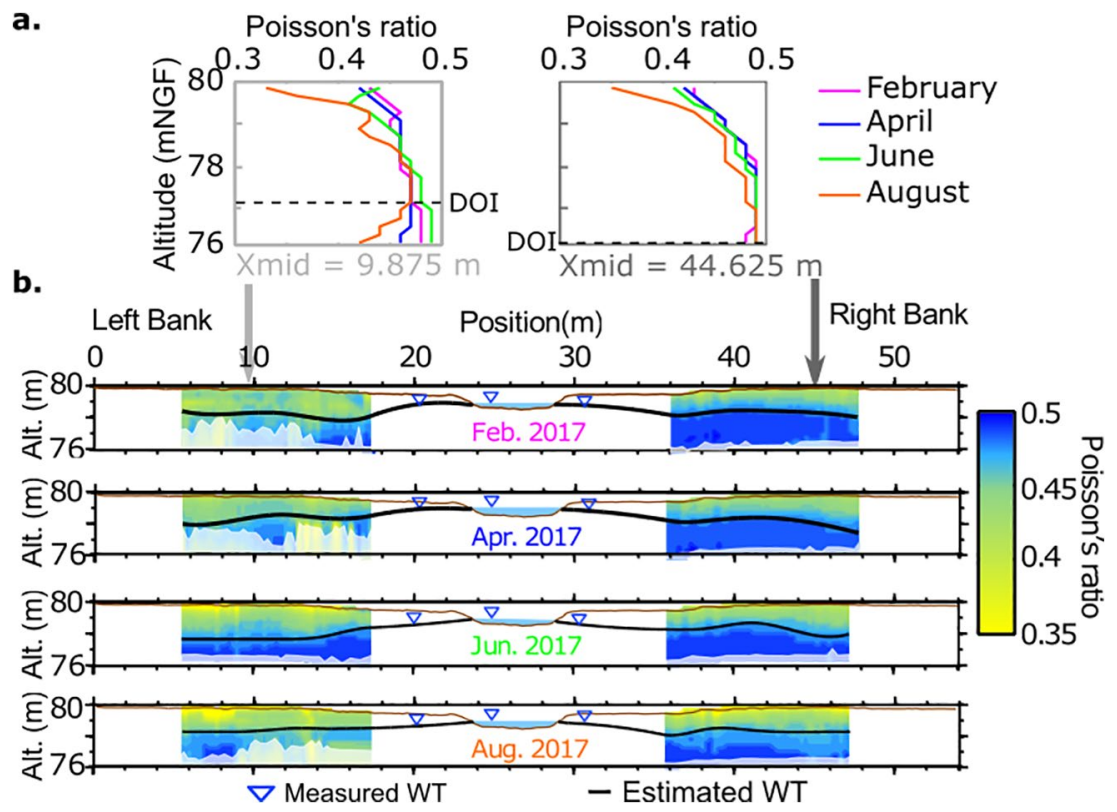

Figure 4. Poisson's ratio $(\nu)$ estimated from seismic data: highest values indicate a saturated medium when low values correspond to partially or unsaturated medium. (a) $1 \mathrm{D} v$ profiles calculated on the left bank (at $9.875 \mathrm{~m}$ ) and the right bank (at $44.625 \mathrm{~m}$ ) for each time step. (b) Pseudo-sections of $\nu$ for each time step and estimated WT models (black lines). The white shaded area indicates depths below the maximum DOI.

tomographic inversion of arrival times (Rohdewald, 2011; Schuster \& Quintus-Bosz, 1993) initialized with a gradient model created by laterally extending a 1D structure derived from the mean of the arrival time and assuming a stratified medium. VS is estimated by 1D inversions of the surface-wave dispersion data collected along the lines (Pasquet \& Bodet, 2017; Socco \& Strobbia, 2004). For each time step, arrival times and dispersion curves were inverted independently. No constraints were imposed on the parametrization, neither in space nor in time, and it remained the same for each time step. Initial models, errors, perturbation evolutions with iteration and final models were systematically controlled and compared for each time step (see details in Dangeard, 2019).

At this stage, it is worth noting that the inversion methods used to estimate velocity models, especially for VS, involved a limited number of layers. Final VP and VS sections (see examples in Appendix A6) were thus projected on a regular grid to compute $v$ as presented in Figure 4. There are no actual conventional rules available yet to systematically estimate neither resolution nor a posteriori uncertainties in near-surface 2D $\nu$ imaging techniques. Even though several strategies exist in seismology and exploration seismics, such as "checkerboard tests" or computation of sensitivity kernels, for instance (e.g., Bauer et al., 2010, 2003), they have not been completely transposed to near-surface applications yet (the combined use of $P$ wave travel time tomography and surface-wave dispersion inversion involve distinct characteristics of the wavefield and different assumptions about the medium, thus providing VP and VS models of different sensitivity, resolution, investigation depth and posterior uncertainties). For these first attempts, the resolution was assumed to be the distance between successive geophones (as, for instance, recommended in Pasquet \& Bodet, 2017; Pasquet, Bodet, Dhemaied, et al., 2015; Pasquet, Bodet, Longuevergne, et al., 2015).

In such porous media, high values of $\nu$ indicate a saturated medium when low values correspond to partially or unsaturated medium. As illustrated by the 1D examples given in Figure 4a, $\nu$ globally increases with depth and shows different saturation gradients depending on the time step. Even if the seismic inversion techniques are limited by their maximum DOI and their resolutions, the obtained $\nu$ profiles then highlight both spatial and temporal evolutions of the saturation along the section. The WT appears to deepen with time between February and August. DOI also provides valuable information. It is computed not only from the measurement setup, but also from the wave paths for VP and the dispersion inversion uncertainties for VS. These criteria are actually linked to the degree of heterogeneity (heterogeneity being itself related to 
3D physical processes far from 1D and 2D assumptions used in the inversion techniques). In Figure 5b, the DOI appears clearly lower on the left bank than in the right bank. The heterogeneities of the lithofacies estimated by the ERT and our knowledge are higher on the left bank than on the right banks. This supports the difference in hydrogeological behavior between banks. The heterogeneities in terms of lithology and therefore hydrodynamic parameters of the left bank are highlighted.

The obtained geometry of $v$ on the pseudo-sections of Figure $5 \mathrm{~b}$ not only shows a global decrease of saturation with time, but also delineates a transition from partially to fully saturated soil. For every horizontal position, $v$ systematically reaches values close to 0.5 and no longer varies with depth. For each time step, among $\nu$ values higher than 0.48 , we then searched for zero values of its gradient with depth. We assumed that above this threshold, the medium was completely saturated. This threshold actually directly comes from a previous study (Pasquet, Bodet, Longuevergne, et al., 2015) performed in the same catchment, area, and lithological context. Pasquet, Bodet, Longuevergne, et al. (2015) estimated the WT depths at different hydrogeological periods, from 1D seismic models in a horizontally layered zone of the site. They validated observed contrasts in $v$ values with both piezometer levels and real water content values from samples collected along a vertical auger sounding profile. However, the inversion methods used to estimate the seismic velocity models involve a limited number of layers projected on a grid, which cannot describe the continuous variations of the water saturation. This is why we decided to interpret it in a binary manner, e.g., non-saturated for low $\nu$ values versus saturated for highest $\nu$ values. This approach has also been verified on $2 \mathrm{D} v$ images obtained in a different context, with verifications from piezometers (Pasquet, Bodet, Dhemaied, et al., 2015).

For each time step in the current study, WT depth values inferred from $\nu$ contrasts were thus spatially interpolated with splines, to better correspond to the continuous variations of this property in the natural environment (using an optimization procedure based on the simulated annealing (e.g., Aarts \& Van Laarhoven, 1985), similar to the algorithm implemented by Maineult (2016) but without its monotonicity criterion. In other words, the total set of depths associated with a value of $\nu$ equal to 0.48 produces a non-continuous curve (NCC), because of the discrete sampling in depth. This cannot reflect the reality of the WT evolution with distance. The spline interpolation, with nodes spaced by a distance of about $3 \mathrm{~m}$, allowed us to obtain a continuous curve (CC), in such a way that the L1 norm between NCC and CC is minimal. We do not have seismic data between 23.75 and $30.25 \mathrm{~m}$ (Figure 2). To bridge this gap, we impose both the RWL and the PLs as constraints of the interpolation. This part of the WT is not used to calibrate the GW model. The depth of the interpreted WT appears more variable on the left bank than on the right bank, linked to the lithological heterogeneities. As a first approach the error in WT depth estimation was considered linked to the resolution of $2 \mathrm{D} v$ sections, that is the distance between successive geophones.

\section{Expanded Stream-Aquifer Interface Model Constrained by Geophysical Results}

\subsection{Expanded Model Setup With Updated Initial and Boundary Conditions}

The multi-method approach described above, enables the simulation of the stream-aquifer exchanges in an expanded model, behind the usual boundaries imposed by the piezometers positions. The 2D finite volume thermo-hydrogeological model Ginette (Rivière, Flipo, et al., 2019; Rivière et al., 2014; Rivière, Jost et al., 2019) is used to simulate the WT evolution. The conceptualization of the unconfined aquifer as described by Rivière, Jost et al. (2019) and Rivière, Flipo, et al. (2019) are employed. The maximal used time step is $900 \mathrm{~s}$. An automatic adjustment of the time step was used during simulations to ensure convergence. The model size is $z=3 \mathrm{~m} \times x=42 \mathrm{~m}$. Vertical and horizontal discretizations are based on empirical resolution from the geophysical methods: a vertical geometric factor of 1.1 and a constant horizontal discretization of $0.25 \mathrm{~m}$ are used. The discretization is finer close to the stream $(3 \mathrm{~cm})$. The domain consists of 2,936 cells.

The hydrodynamic parameters, permeability and porosity, are assigned to each lithofacies depicted in Figure 3b. The flow modeling covers the time period from February 13, 2017 to August 31, 2017. The estimation of the WT in February 2017 is used as the initial conditions. A 10 day spin-up period with transient conditions is used to ensure the transient behavior of the initial state of the domain. The boundary conditions are: (a) no-flow Neumann boundary condition is prescribed at the bottom of the model, considering 
a.

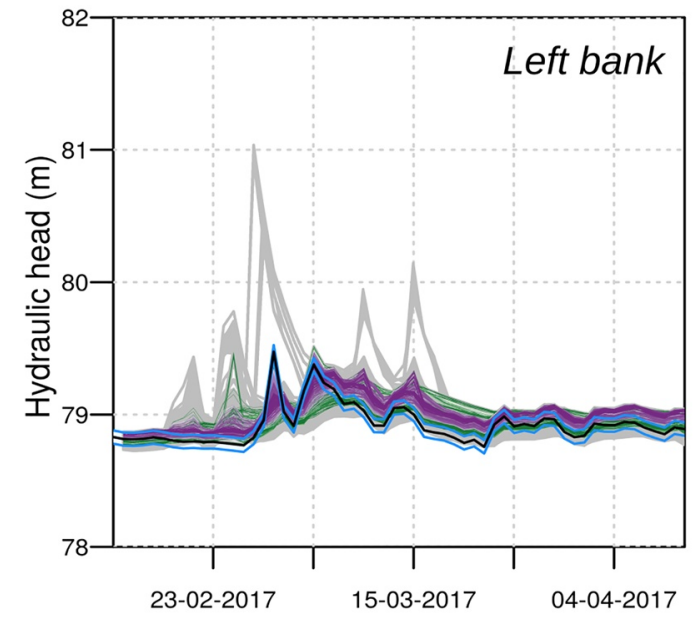

b.

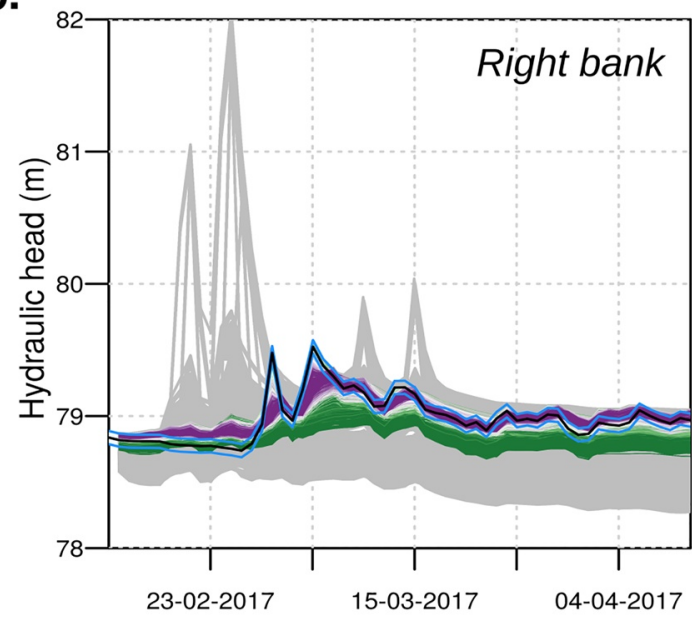

c.

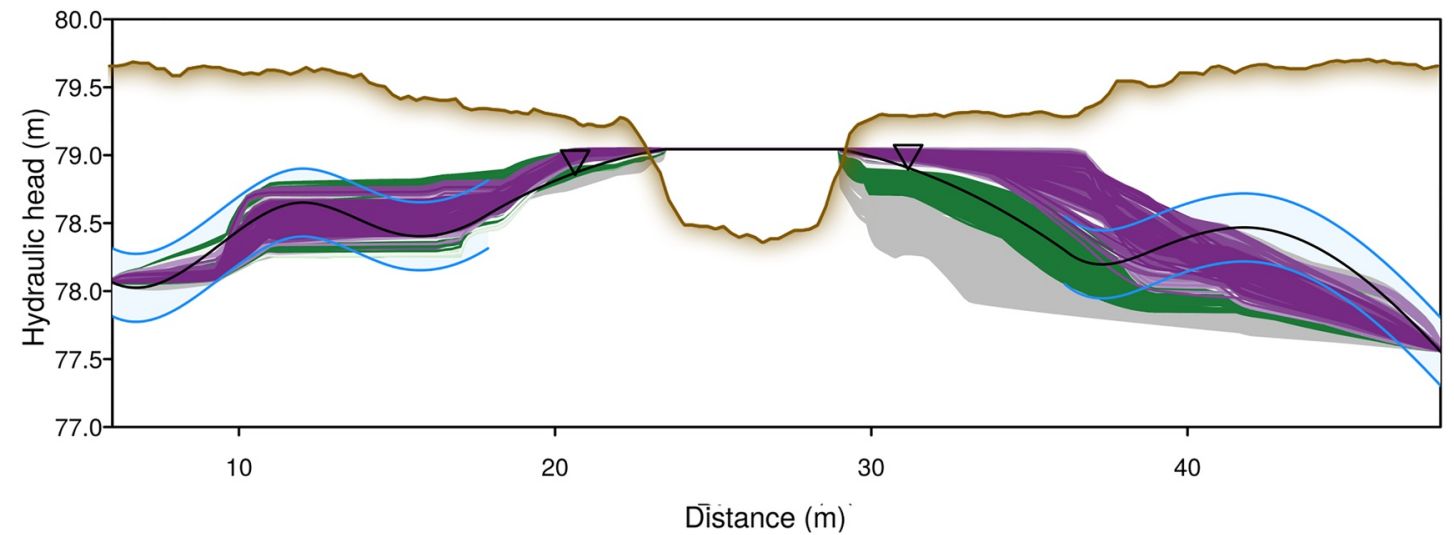

d.

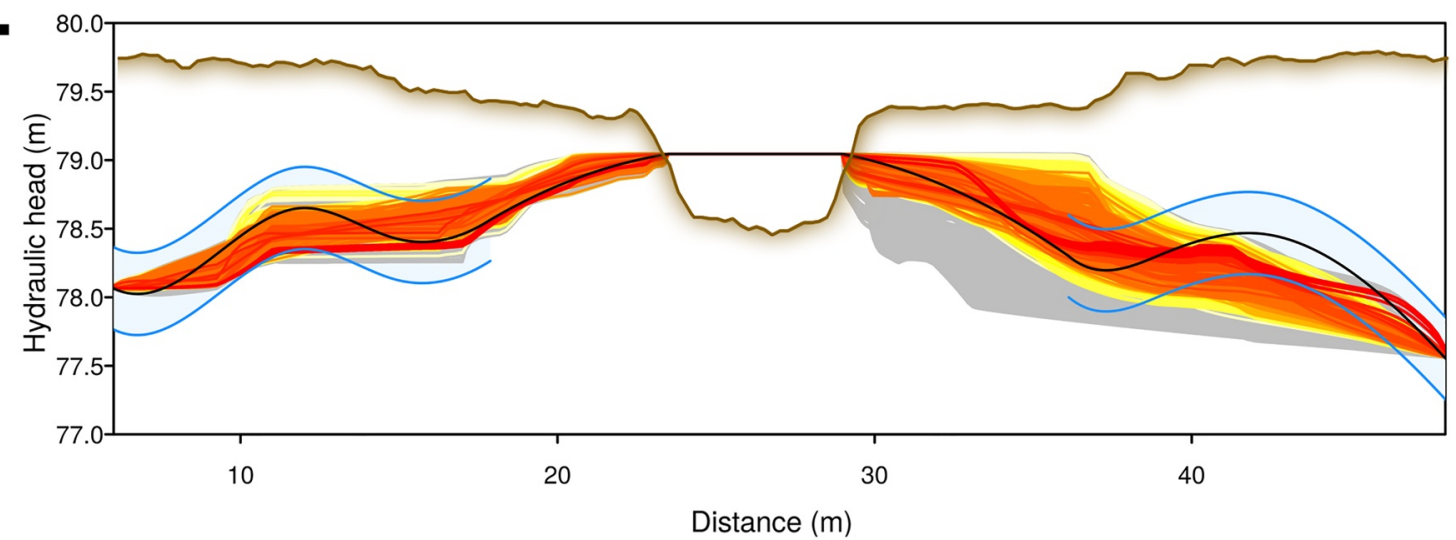

Piezometer position

All simulation

- Ground surface

Seismic WT model and hydraulic head within errors

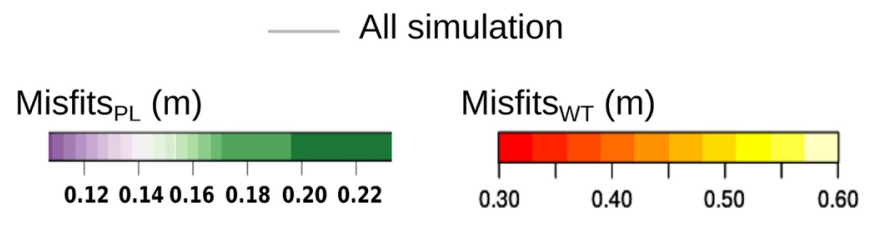

Figure 5. Screening parameters of simulated PL time series on both left (a) and right (b) banks compared to the observed PL during the calibration period. The errors ( $5 \mathrm{~cm}$ here) correspond to the sum of the uncertainties of the pressure sensor, the barometric measurements, the length of the cable, and the georeferencement of the piezometer. (c) WT along the cross-section simulated during the screening compared to the WT estimated from seismic models. The errors were defined, as a first approach, as equivalent to the seismic method resolution $(0.25 \mathrm{~m}$, i.e., the distance between successive geophones). 
the very low permeability of the underlying marl; (b) transient Dirichlet boundary conditions corresponding to the RWL time-series measured are applied at the top of the river meshes; (c) the effective rain is imposed at the soil surface, calculated from rainfall and evapotranspiration data (Tallec et al., 2015) assuming zero runoff and (d) each edge boundary condition is provided by a linear interpolation in time of seismic interpretations.

\subsection{Model Calibration and Validation}

The eight hydrofacieses of the domain are calibrated during the period between February and April. The calibration was performed on the geophysical estimation of the WT in April and the fluctuation of the PL measured in the banks during 57 days with the model output. There are 57 data for each PL and 295 data for WT. Because of the difference in the nature of the observations despite their common unit, it is to choose a weight, as mentioned by Schilling et al. (2019). Model is calibrated by the use of a parameter-sampling script to minimize two misfits:

1. The misfit $_{\mathrm{PL}}$ between the simulated and observed PL time series

$$
\operatorname{misfit}_{\mathrm{PL}}=\sqrt{\frac{1}{N} \sum_{i=1}^{N}\left(d_{\mathrm{RB}}^{i}-s_{\mathrm{RB}}^{i}\right)^{2}}+\sqrt{\frac{1}{N} \sum_{i=1}^{N}\left(d_{\mathrm{LB}}^{i}-s_{\mathrm{LB}}^{i}\right)^{2}},
$$

with $d_{\mathrm{RB}}^{i}$ and $s_{\mathrm{RB}}^{i}$ respectively the observed and simulated right bank PL (m) at each i-day during the whole period ( $\mathrm{N}$ data), $d_{\mathrm{LB}}^{i}$ and $s_{\mathrm{LB}}^{i}$ the same data on the left bank (m),

2. The misfit $_{\mathrm{WT}}$ between the simulated and observed WT through the best simulation of the PL

$$
\left(\text { misfit }_{P L}<0.25\right)
$$

$$
\text { misfit }_{\mathrm{WT}}=\sqrt{\frac{1}{M} \sum_{j=1}^{M}\left(d_{\mathrm{WT}}^{j}-s_{\mathrm{WT}}^{j}\right)^{2}},
$$

with $d_{\mathrm{WT}}^{j}$ and $s_{\mathrm{WT}}^{j}$ respectively the observed and simulated WT (m) in April along the seismic sections (M data).

WT and PL provide, respectively, spatial and temporal information for model calibration. The misfit ${ }_{\mathrm{WT}}$ is calculated for the simulations whose the misfit ${ }_{\mathrm{PL}}$ is less than $0.25 \mathrm{~m}$. This methodology allows us to discriminate against the best parameters setup. Using the script for parameter sampling, the hydraulic conductivity $(\mathrm{K})$ varies from $10^{-8}$ to $10^{-3} \mathrm{~m} \cdot \mathrm{s}^{-1}$ (clay/silt to sand/gravel) and the porosity $(\omega)$ from 0.001 to 0.2 .

The misfit values for the time series piezometers are between 0.006 and $0.65 \mathrm{~m}$ for the right bank, and between 0.005 and $0.20 \mathrm{~m}$ for the left bank over the calibration period. For the spatial variation of the WT, misfits are between 0.09 and $10.6 \mathrm{~m}$. The calibration result is shown in Figure 5. The lower values of the piezometer misfits (depicted in purple and green) correspond to the simulations with a misfit ${ }_{\mathrm{PL}}$ lower than $0.25 \mathrm{~m}$. Therefore, the piezometer times series do not contain sufficient information to find an appropriate parametrization to reproduce the estimated WT with the time-lapse seismic. The misfit ${ }_{\mathrm{wT}}$ allows us to discriminate the parameters setup. The 2,209 over 206,428 simulations get a cumulative misfit value below $0.25 \mathrm{~m}$.

The parameterization was then verified for a 7 month simulation (February 2017 to August 2017) against the PL at the right and left banks and the WT estimated in April, June, and August (Figure 6). The depicted misfit is the sum of the root mean square:

$$
\text { misfit }_{\text {sum }}=\sqrt{\frac{1}{N} \sum_{i=1}^{N}\left(d_{\mathrm{RB}}^{i}-s_{\mathrm{RB}}^{i}\right)^{2}}+\sqrt{\frac{1}{N} \sum_{i=1}^{N}\left(d_{\mathrm{LB}}^{i}-s_{\mathrm{LB}}^{i}\right)^{2}}+\sqrt{\frac{1}{M} \sum_{j=1}^{M}\left(d_{\mathrm{WT}}^{j}-s_{\mathrm{WT}}^{j}\right)^{2}},
$$

The model simulations correctly reproduce the temporal and spatial variations of the WT (Figure 6) using plausible calibrated parameters which are compared to prior values as shown in Table 1. The calibrated porosities are lower than prior values which could be due to the considered scale and to the non-unicity of the problem implying equivalencies for various (porosity, permeability) couples. 
a.

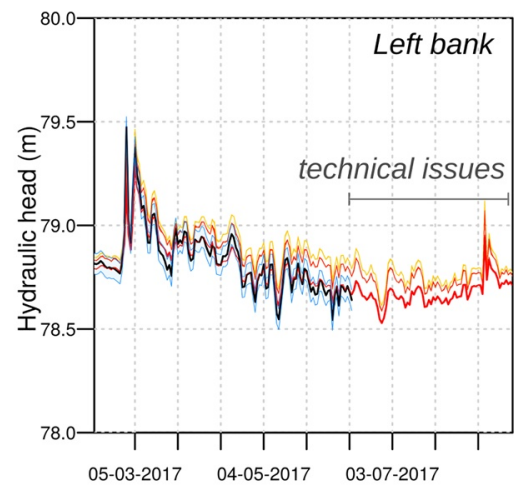

b.

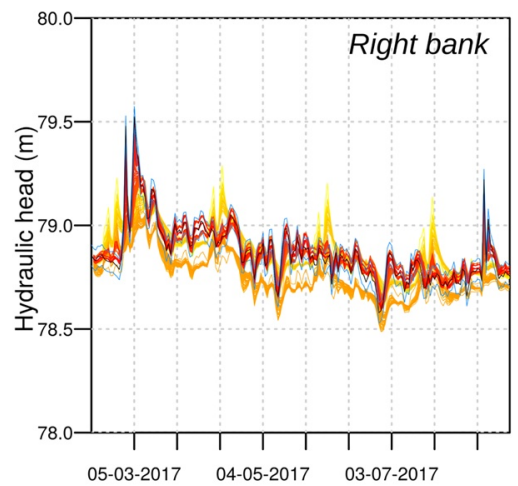

C.
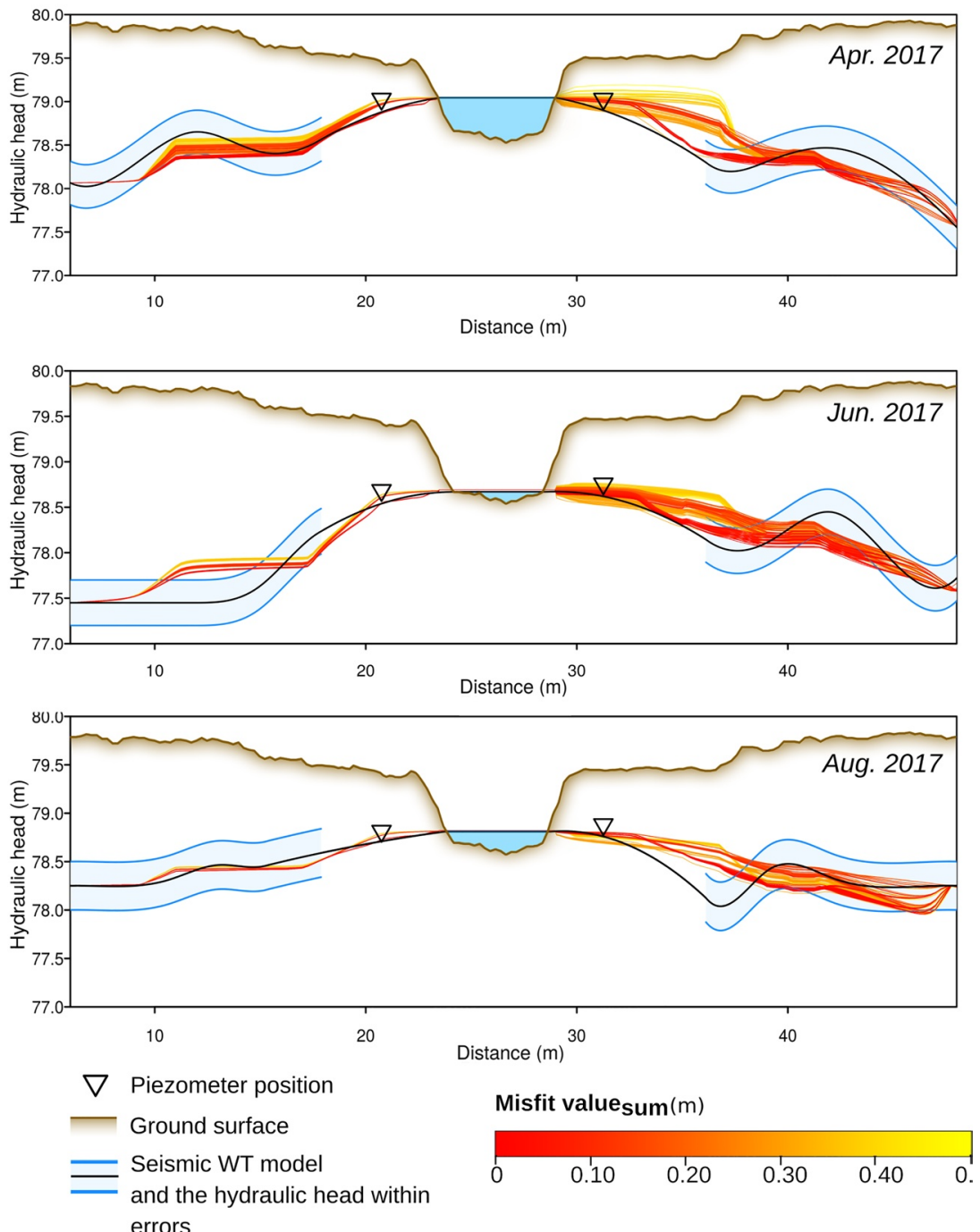

Figure 6. Screening parameters results (with colors depending on misfit value) during the calibration period on both left (a) and right (b) banks compared to the observed PL. The errors ( $5 \mathrm{~cm}$ here) correspond to the sum of the uncertainties of the pressure sensor, the barometric measurements, the length of the cable and the georeferencement of the piezometer. (c) WT simulated along the domain and compared (colors depending on misfit value) to WT seismic models (black lines) within errors (blue lines). The errors were defined, as a first approach, as equivalent to the seismic method resolution $(0.25 \mathrm{~m}$, i.e., the distance between successive geophones). 
Table 1

Hydrodynamic Parameters Used in the Numerical Simulations

\begin{tabular}{|c|c|c|c|c|}
\hline \multirow[b]{2}{*}{ Hydrofacies } & \multicolumn{2}{|c|}{ Hydraulic conductivity, $\mathrm{K}\left(\mathrm{m} . \mathrm{s}^{-1}\right)$} & \multicolumn{2}{|c|}{ Porosity, $\omega(-)$} \\
\hline & A priori values & Calibrated values & A priori values & Calibrated values \\
\hline Agricultural soil (1) & $110^{-5 b}$ & $110^{-5}$ & & 0.2 \\
\hline Silt (2) & $310^{-7 a}$ & $110^{-4}$ & $0.13^{\mathrm{a}}$ & 0.02 \\
\hline Overbank (silt, sand) (3) & $210^{-4}-1.910^{-5 a}$ & $110^{-4}$ & $0.265-0.31^{\mathrm{a}}$ & 0.01 \\
\hline Channel lag deposit (gravel, coarse sand) (4) & $2.910^{-3 a}$ & $110^{-3}$ & $0.25^{\mathrm{a}}$ & 0.04 \\
\hline Levee (silt) (5) & $1.910^{-5 a}$ & $110^{-5}$ & $0.265^{\mathrm{a}}$ & 0.01 \\
\hline Marl (6) & $310^{-6 c}$ & $110^{-6}$ & $0.0015-0.002^{\mathrm{c}}$ & 0.075 \\
\hline Green clay (7) & $210^{-7 \mathrm{c}}$ & $110^{-7}$ & $0.015^{\mathrm{c}}$ & 0.05 \\
\hline Streambed (8) & $310^{-5 c}$ & $110^{-5}$ & $0.1^{\mathrm{a}}$ & 0.2 \\
\hline
\end{tabular}

${ }^{a}$ Morris and Johnson (1967). ${ }^{\mathrm{b} B a t k o v a}$ et al. (2020). 'Berrhouma (2018) for the same location.

The simulations of the PL and the WT geometry in June and in August prove the relevance of the selected hydrodynamics parameters considering the expanded domain and the extrapolated boundary conditions (Figure 6). In addition, the seismic interpolation of the WT provides the opportunity to check the WT geometry in April, June, and August. On the left bank, the oscillations of the WT due to the heterogeneity previously described are correctly reproduced with the best fit (i.e., the lower misfit value) occurring in August, at the last time step. In June, the discrepancy between the simulated and the interpolated WT is actually due to a storm event that occurred after the seismic acquisition for this bank. On the contrary, the acquisition was performed during the rain on the right bank which behavior is correctly simulated. The ERT static characterization along the cross-section shows the presence of two contrasts of hydraulic conductivity clearly influencing the geometry of the WT. However, the WT inflection point is shifted in space $(2.5 \mathrm{~m})$ at positions $x=12 \mathrm{~m}$ and $x=42 \mathrm{~m}$ (Figure 6). These issues are due to difficulty in estimating a posteriori uncertainties in the resistivity models with the ERT method used in the current study (Figure 3a). To improve the calibration, the geometry of the hydrofacies should be slightly improved with associated uncertainties to be included in the simulations (e.g., using clustering techniques, Sabor et al., 2021).

\subsection{Flow Pathway Patterns}

The simple model simulates the water flow evolution until the pressure sensor of the left banks stops functioning, while the river corridor model is used to perform simulations during the whole period, using the conditions interpolated thanks to the estimated WT. This is the first advantage of our methodology. The river corridor model forms preferential flow paths if compared to the simple, as shown in Figure 7 . The velocities simulated with the homogeneous model are low $\left(1.10^{-9}-1 \cdot 10^{-7} \mathrm{~m} \cdot \mathrm{s}^{-1}\right)$. The simple model simulates the GW divide at the center of the river and almost symmetrical flow paths. The river corridor model simulates asymmetrical flow conditions and the deformation of GW divide with the time. Local seepage rates along the SW-GW interface were found to be highly variable in space and time both within and among the river corridor models. The most important exchanges SW-GW are found in the left banks with velocity of $1.10^{-7}-1.10^{-5} \mathrm{~m} . \mathrm{s}^{-1}$. This part of the SW-GW stays in downwelling configuration even after the flood events. Spatial variability was mainly governed by the distribution of hydrofacies and the corresponding hydraulic conductivity which are calibrated thanks to the WT geometry estimation. In addition, the simulations show that the heterogeneous model shows reversal flow path in comparison to the homogeneous model below the streambed. Unfortunately, the simple model does not manage to capture the flow pattern occurring in the aquifer. The thermal profiles in the hyporheic zone are not located in the left heterogeneities. This proves that the use of the heat as the tracer reflects only local processes. Using time-lapse seismics, it was possible to image the WT for each time step. This key information provides the inputs making it possible to simulate the flow path way. In general, the estimation of the flow path is given by the use of methodology covering larger areas and lacking resolution, such as artificial tracers (dye tracers), natural tracers (heat tracers and hydrochemistry data, stable isotopes (e.g., $\delta^{2} \mathrm{H}$ and $\left.\delta^{18} \mathrm{O}\right),{ }^{222} \mathrm{Rn}$, dissolved atmospheric gases), or 

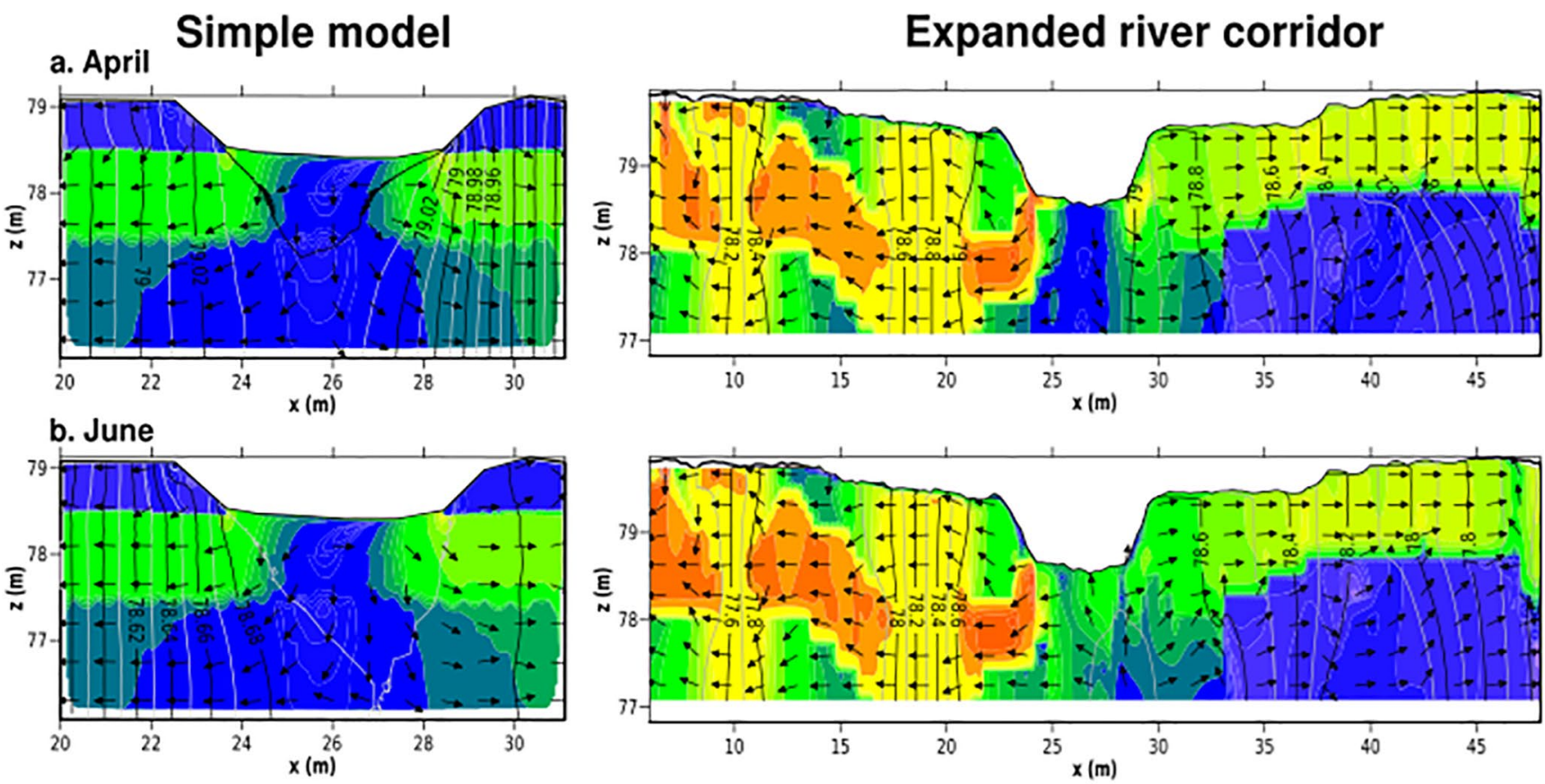

\section{c. August}

$\rightarrow$ Flow direction

-78.6- Hydraulic head (m)
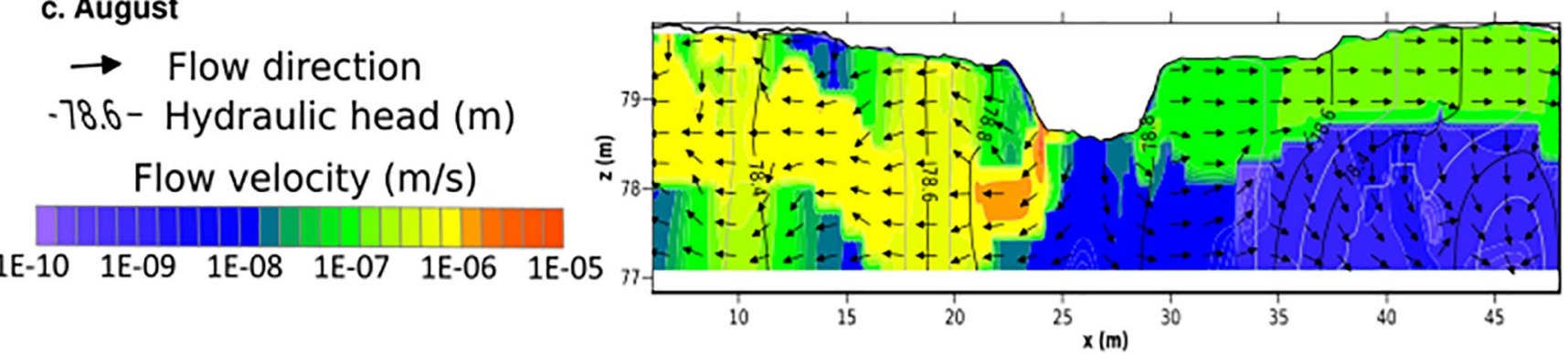

Figure 7. Head and flow velocity distribution: Panel (a) for the simulation obtained in the homogeneous case (simple model) and Panel (b) the simulation of the heterogeneous case (expanded model). The arrows represent the flow directions.

tracers of anthropogenic origin including persistent organic micropollutants and dating methods $\left({ }^{3} \mathrm{H} \mathrm{Cl}^{-}\right.$, $\mathrm{Br}^{-}, \mathrm{CFCs}, \mathrm{SF}_{6}$ and ${ }^{3} \mathrm{H} /{ }^{3} \mathrm{He}$ ). These methods could nevertheless be used to validate the flow path at the site scale, but not with the resolution of our river corridor model.

The calibration of distributed hydrodynamic parameters along the river corridor domain, checked with time-lapse snapshots of the WT geometry, eventually enables the quantification of local stream-aquifer exchanges (Figure 8). The results show that the river-aquifer exchanges respond rapidly to the river discharge change in Figure 8. The infiltration rate simulated by Berrhouma (2018) is in the same order of magnitude of those simulated with the river corridor model. Therefore, the same exchange rate can come from different flow paths patterns and different hydrofacies fields. The water quality is largely influenced by the time that a water parcel stays in contact with the reactive media and by the mixing ratio of SW and GW as it is demonstrated by Shuai et al. (2017). Therefore, in the different model setups, the water quality will differ.

\subsection{Response SW-GW Interactions to Flood Events}

The responses of the SW-GW exchanges to a flood event change between the homogenous model and the river corridor model. For the river corridor model, the infiltration follows a sequence. First, the river discharge into the aquifer increases when the flood peak arrives. Second, the river drains the riparian zone during the recession period. This sequence is also simulated with the homogeneous model but only for the major flood event and the resulting velocities are lower. For the other event, the total net SW-GW infiltra- 

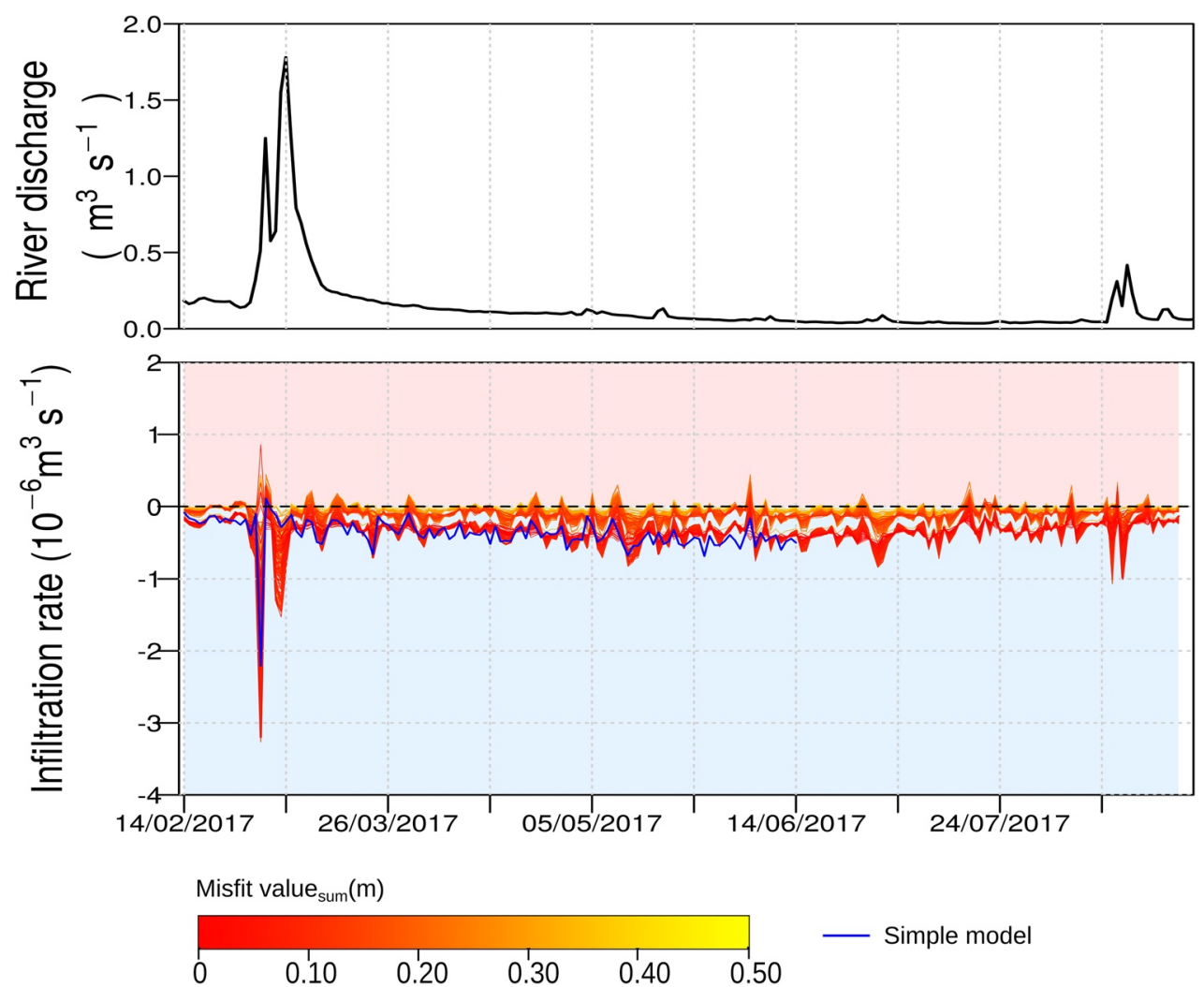

Figure 8. Simulated stream-aquifer exchanges through time. Negative values indicate fluxes from the stream to the aquifer while positive ones are from the aquifer to the stream.

tion stays in downwelling configuration because the hydraulic conductivity is low. This pattern of SW-GW exchange will have significant impacts on biogeochemical processes in the hyporheic zone during the flood event (Figure 9).

\section{Conclusions}

River corridor systems have a heterogeneous sedimentary structure that results from stream processes (including channel meandering history) and contains significant preferential flow paths. These flow paths are crucial to determine the water residence time and biogeochemistry fluxes. Different methodologies have been developed in the last decades to quantify the SW-GW exchanges but none allows to get the flow path. A novel modeling framework was developed that enabled describing the flow paths within the river corridor and ensuring their potentiality with the geometry of the WT. The combination of ERT, time-lapse seismic, geotechnics, and hydrogeological modeling presented in this study enables: (a) the mapping of the hydrofacies along both studied banks; (b) the delineation of the WT depth in space and time, (c) expansion of the hydrogeological modeling domain beyond the usual boundaries and (d) the characterization of the flow paths within the river corridor. As for initial conditions, a common practice consists in specifying them as either arbitrary or observation-based by running a steady-state model, despite the widely recognized persistence of the initial steady state. Here, the time-lapse seismic acquisition makes it possible to specify the initial state (e.g., initial hydraulic head) of the hydrological model. As always in hydrological modeling frameworks, the problem of equifinality arises (Beven, 2006). Reducing the ill-posedness of flow models by introducing additional and unconventional observations into the calibration process thus allows artificially reducing the model complexity by simplifying the geometry or homogenizing parameters of hydrofacies (Schilling et al., 2019). The time-lapse seismic observations not only provide WT snapshots, but also help calibrating the hydrofacies and by this way, describing the flow paths on a 2D expanded section. Future 
a. 28-02-2017

\section{Simple model}

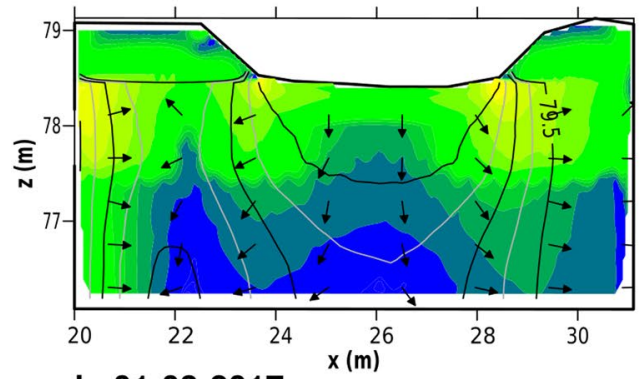

b. $01-03-2017$

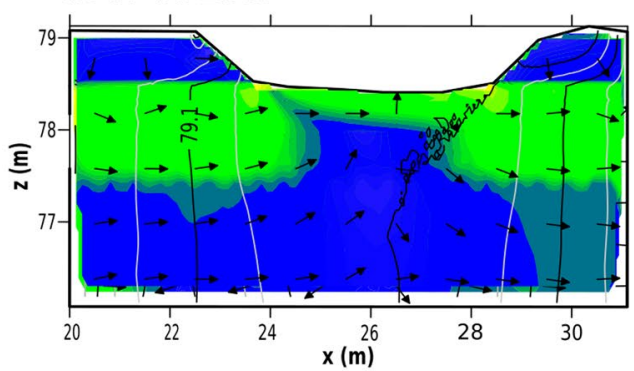

$\rightarrow$ Flow direction -78.6- Hydraulic head (m)

\section{Expanded river corridor}
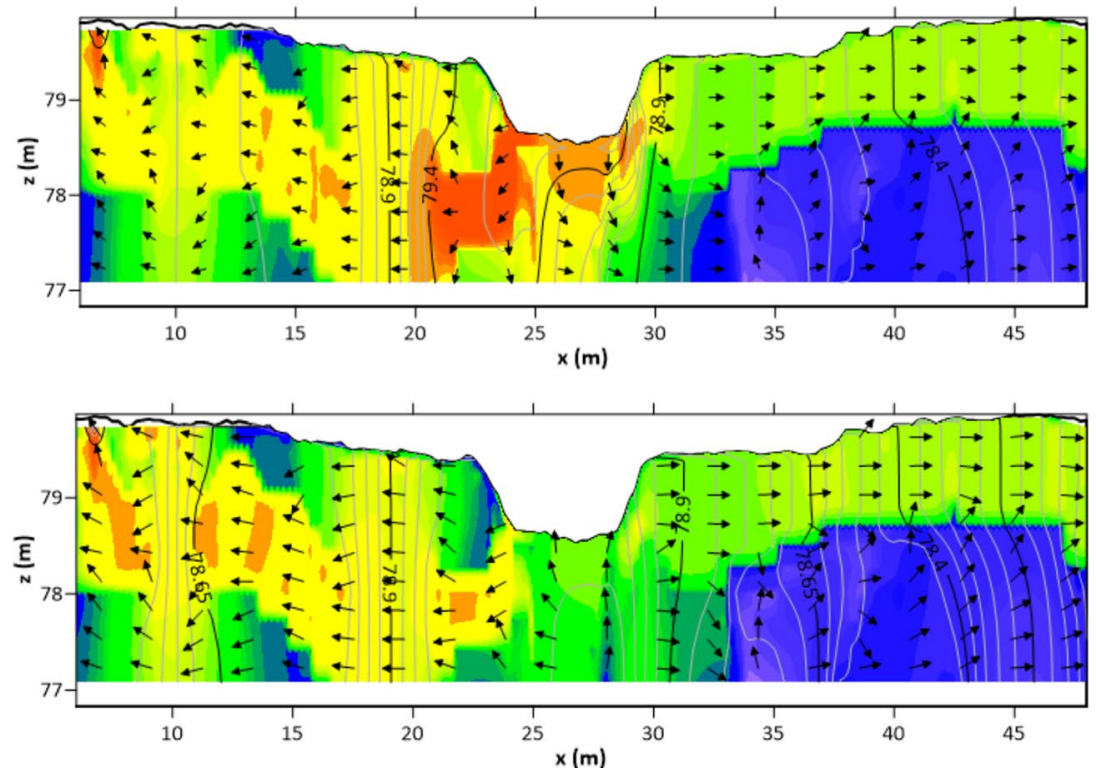

Flow velocity $(\mathrm{m} / \mathrm{s})$

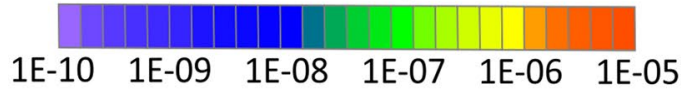

Figure 9. Head and flow velocity distribution during the major flood event: Panel (a) for the simulation obtained in the homogeneous case (simple model) and Panel (b) the simulation of the heterogeneous case (river corridor). The arrows represent the flow directions.

investigations may include using different tracers such as ${ }^{37} \mathrm{Ar},{ }^{222} \mathrm{Rn}$ or ${ }^{3} \mathrm{H} /{ }^{3} \mathrm{He}$ (Pardo-Alvarez et al., 2018) in order to comfort the global behavior of the flow paths.

On the one hand, the methodology presented in this study can be developed at the larger scale in order to understand the global flow directions as well, more particularly more important seismic sensors networks can be considered. On the other hand, it can also be used at a reduced scale with higher coverage and resolution. The seismic data analyses could be pushed further by determining the unsaturated zone parameter thanks to $\nu$ or derived parameters. To do so, specific petrophysics (Jougnot et al., 2015; Rubin \& Hubbard, 2006) must be explored to better link hydrogeological parameters to geophysical data in a quantitative way, yielding toward a coupled hydrogeophysical modeling framework (e.g., Hubbard \& Linde, 2011). Blazevic et al. (2020) recently proved that the link between seismic observable and petrophysical predictions are promising to improve the inversion with a good knowledge of the soil's properties. $v$ is an elastic property that can be used to describe the stress state variables of porous media. A first step would be to obtain a relationship between the saturation degree and $\nu$ by using laboratory experiments. Some additional measurements would be needed to better constraint the relationship such as the porosity and the saturated hydraulic conductivity. On the field, the next step would be to take advantage of distributed temperature sensing. The temperature profiles will increase the constraint of the hydrofacies calibration (hydraulic conductivity, porosity, and unsaturated parameters) but also the evolution of the unsaturated zone which limits the heat conduction. These future results can provide structural information about the saturation profiles and help quantitatively inverting seismic data by joint inversion. The hydrological model would then take into account the effects of the partially saturated area, strongly linked to the WT oscillations and shallow GW recharge. 


\section{Appendix A: Geophysical Data Processing and Interpretation}

\section{Appendix A1. ERT Setup and Data}

As mentioned in Section 3, a 0.25 m spaced 72-electrode Wenner-Schlumberger array was deployed on the left bank, perpendicular to the river (Figure A1). The right bank, for its part, left room for a 96-electrode setup (same spacing, same configuration, and same direction). On the left bank, at least 1,318 apparent resistivity values are measured. The injected current was between 32.65 and $263.8 \mathrm{~mA}$, minimum measured electrical potentials were 29.06 and $10,660 \mathrm{mV}$ and standard deviation is $0.147 \mathrm{mV}$. On the right bank, at least 1,107 apparent resistivity values were measured. The injected current was between 37.56 and $220.7 \mathrm{~mA}$, minimum measured electrical potentials were 215.21 and 7,681 mV and standard deviation is $0.195 \mathrm{mV}$. Resulting apparent resistivity pseudosections are presented in Figure A2.

ESE Avenelles

WNW

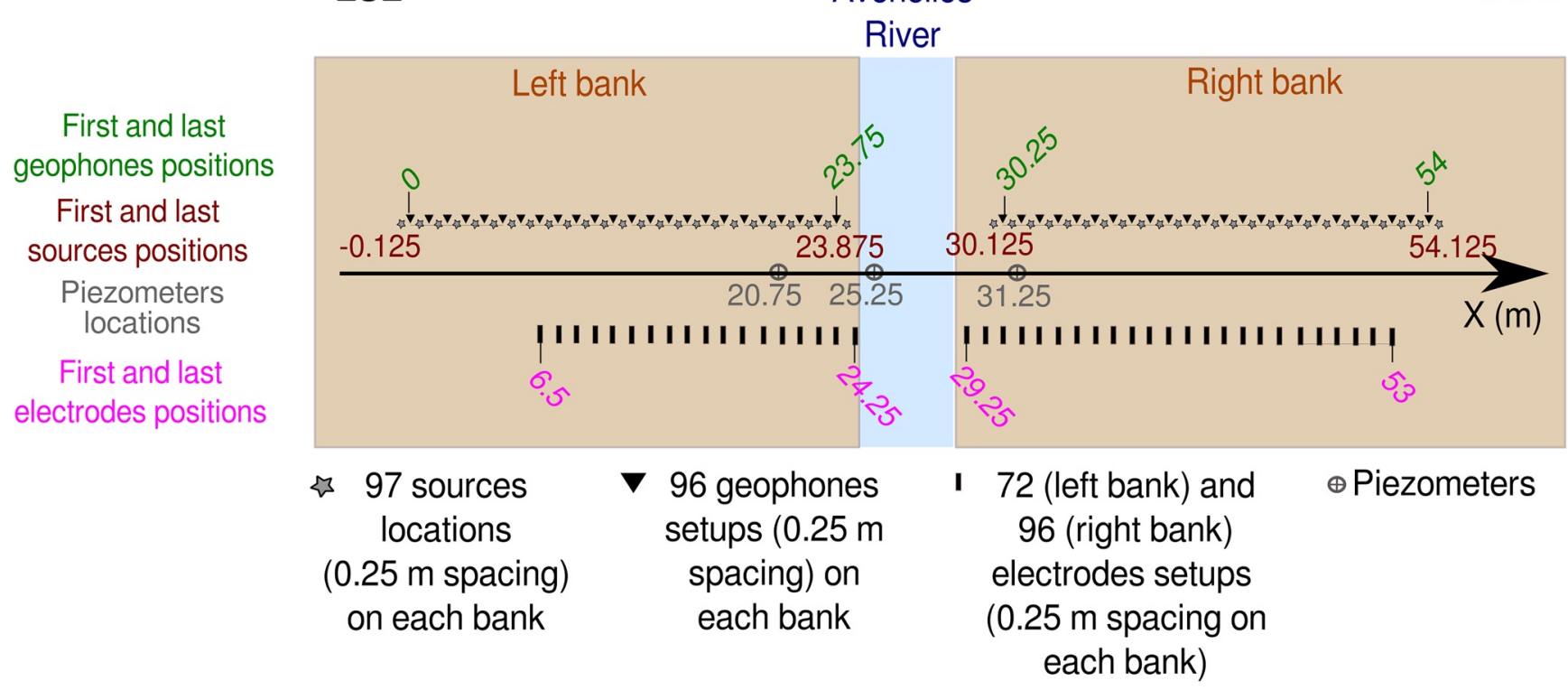

Figure A1. Schematic of acquisition setups deployed to perform seismic (top) and resistivity (bottom) measurements (figure is not to scale; sources, geophones, electrodes, and piezometers were located along the same line; their location are given in meters, the origin being the position of the first geophone on the left bank).

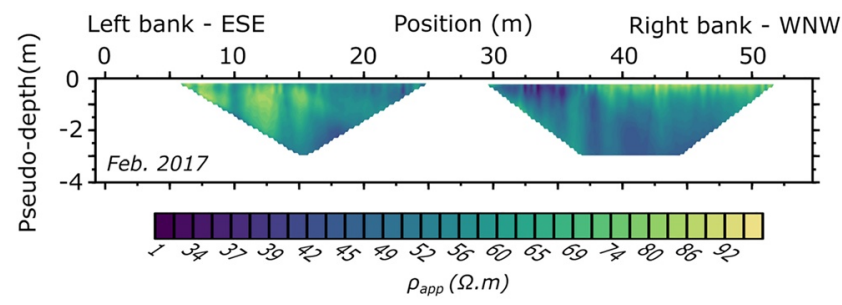

Figure A2. Apparent resistivity pseudosections recorded along both ESE-WNW profiles perpendicular to the Avenelles river.

Data sets were of good quality (i.e., with very low standard deviations). Inversions were performed with Res2dinv (version 3.54.44, Loke \& Barker, 1996) using the standard constraint option (norm L2) which tends to produce models with the smooth boundary between different regions (because the contrast in resistivity was weak, and the signal-to-noise ratio was low). The sections converged after four iterations with relatively low RMS error. ERT sections are presented in Section 3. 


\section{Appendix A2. Dynamic Cone Penetrometer (DCP)}

DCP tests using PANDA2 $\subseteq$ were realized along the profile (Benz-Navarrete, 2009) to highlight subsurface heterogeneities. Tests were done using a $2 \mathrm{~cm}^{2}$ cone. The maximum depth is $1,25 \mathrm{~m}$ (as the rod had to be manually removed, and because of suction effects, we could not go deeper). We defined a refusal after 5 manual blows without sinking. Figure A3 shows soil resistivity values as a function of depth.
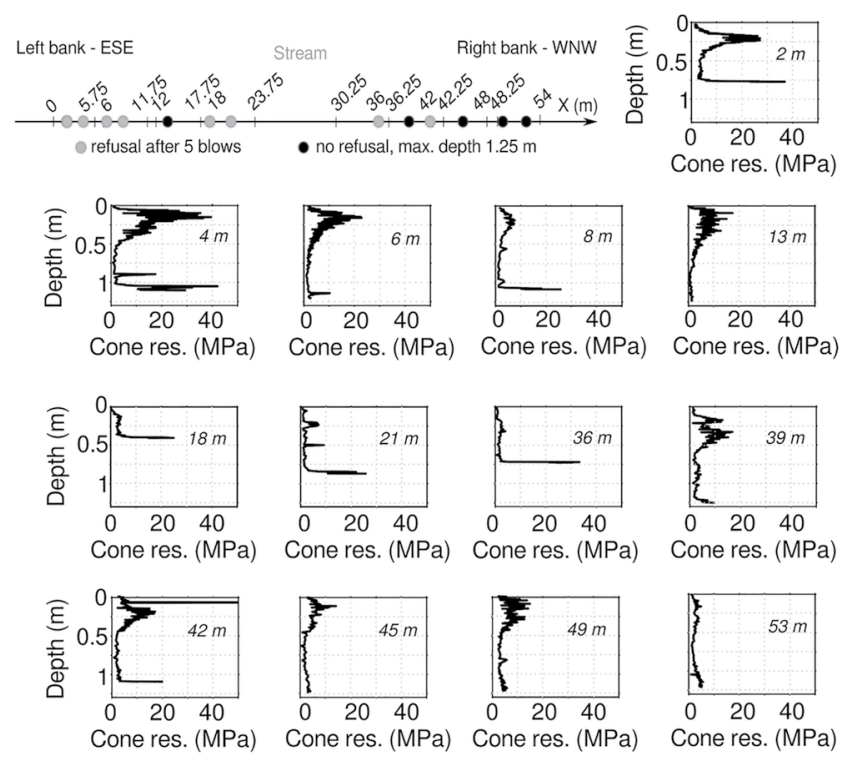

Figure A3. Penetrograms obtained along the SE-NW profile (the inset values on each subplot are the positions along the lines). A $2 \mathrm{~cm}^{2}$ cone was used. A refusal is considered after 5 blows without sinking.

\section{Appendix A3. Time-Lapse Seismic Setup and Processing}

As mentioned in Section 4, a $23.75 \mathrm{~m}$ long profile was deployed on each bank, using a 96-channel seismic recorder with $14 \mathrm{~Hz}$ vertical component geophones $0.25 \mathrm{~m}$ spaced. The setup is detailed in Figure A1. The source was a metal plate hit vertically with a $1.25 \mathrm{~kg}$ hammer. A total number of 97 shots were made along each line with a $0.25 \mathrm{~m}$ spacing starting $0.125 \mathrm{~m}$ before the first geophone. At each position along the lines, we recorded and stacked 6 seismograms in the time domain to increase the signal-to-noise ratio. The sampling rate was $0.5 \mathrm{~ms}$ and the recording length was $2 \mathrm{~s}$, including a pre-trigger delay of $-0.02 \mathrm{~s}$, to record the full surface-wave trains, coda, and background noise. The raw seismic data eventually consists of a total number of 97 seismograms per bank at each time step $(97 \times 2 \times 4=776$ seismograms in total), to be interpreted for velocity models (e.g., Pasquet \& Bodet, 2017). Two seismograms are given as examples in Figures A4a and A4b. Picked $P$ wave first arrival times (in red on Figures A4a and A4b) and surface-wave dispersion curves (in white on Figures A4c and A4d) are shown as well as examples of the actual data of interest to this study. 


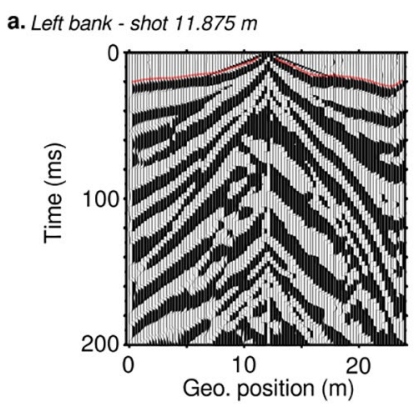

b. Right bank - shot $42.125 \mathrm{~m}$
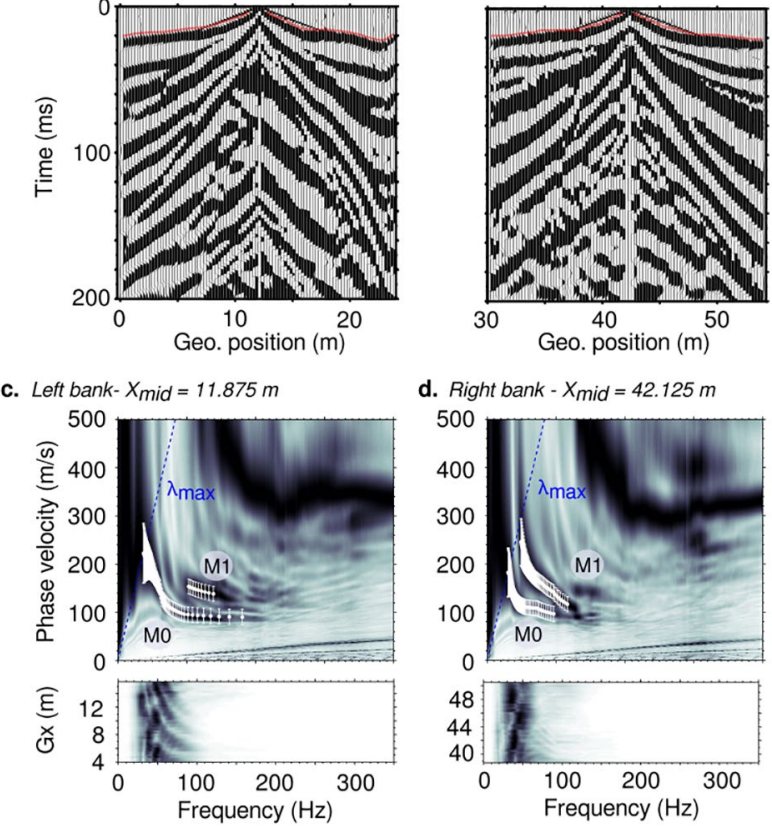

d. Right bank $-X_{\text {mid }}=42.125 \mathrm{~m}$
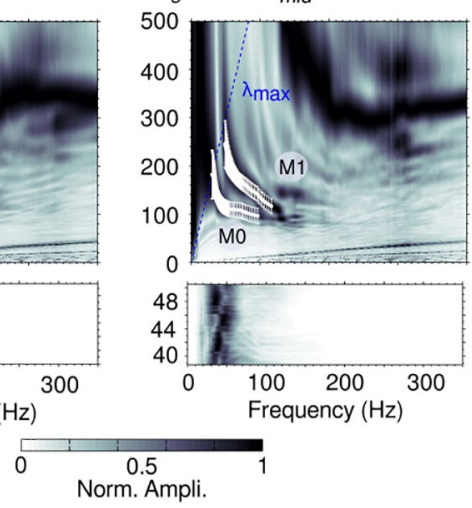

orm. Ampli.

Figure A4. (a) Recorded seismograms for two example shots at $11.875 \mathrm{~m}$ and (b) at $42.125 \mathrm{~m}$ for February 2017 acquisitions. The vertical wiggles represent particle vertical displacement velocities recorded at each geophone through time. The red lines indicate manually picked arrival time values. (c) Example of dispersion images (top) and spectrograms (bottom) obtained at positions $11.875 \mathrm{~m}$ and (d) $42.125 \mathrm{~m}$ (Xmid being the center of the stacking window) for February 2017 acquisitions. The white dots correspond to surface-wave fundamental (M0) and first higher modes (M1), manually picked within errors and below Lmax, the maximum interpretable wavelength.

Pressure waves can be easily identified and their associated first arrival times were manually picked (red lines on Figures A4a and A4b) for the 97 shots on each bank, at each time step, following the workflow described in details in Dangeard et al. (2018). The picked data indicate an apparent velocity increase with depth and do not show marked contrast along the lines apart from topographic effects (more details about $P$ wave data processing and interpretation can be found in Bauer et al., 2010; Bergamo et al., 2016a, 2016b; Pasquet, Bodet, Dhemaied, et al., 2015; Pasquet, Bodet, Longuevergne, et al., 2015). As for surface waves, local phase velocities were extracted along the line, based on the basic methodology of multichannel analysis of surface wave (O’Neill, 2003; Park et al., 1999; Socco \& Strobbia, 2004), yet using windowing and dispersion stacking techniques, following the workflow designed by O'Neill et al. (2003) and implemented by Pasquet and Bodet (2017). Results provided by direct and reverse shots were first extracted separately compared to check the possible influence of strong lateral heterogeneities, as recommended by Bodet et al. (2005), and to define the optimal window size. A good trade-off between lateral resolution and DOI of the method was found with a $11.75 \mathrm{~m}$ wide stacking window, including 8 reverse shots and 8 direct shots. The dispersion curves were manually extracted along the line, rejecting wavelengths greater than $12 \mathrm{~m}$ as recommended by (Bodet et al., 2005, 2009). The first window was centered at Xmid $5.875 \mathrm{~m}$ and the last one was at Xmid $17.875 \mathrm{~m}$ for the left bank and centered at 36.125 and $48.125 \mathrm{~m}$ on the right bank, providing a total number of 98 window positions along each bank, at each time step (see examples for two positions in Figures A4c and A4d). Up to 4 propagation modes could be observed and identified as fundamental (M0), first, second, and third higher modes. Only M0 is considered as a reference for time-lapse observations here, as recently suggested (Bergamo et al., 2016b; Bergamo \& Socco, 2016; Dangeard et al., 2018; Pasquet, Bodet, et al., 2016) but every mode is included in the inversion process. 


\section{Appendix A4. Estimating Seismic Data Picking Errors and Absolute Differences}

In order to properly invert this data set at each time step and discriminate their time-lapse variations from noise or measurement errors, a thorough statistical analysis had to be performed, following the workflow provided by Dangeard et al. (2018). Five shot positions (for arrival times) and window centers (for surface wave dispersion curves) were selected and manually picked 15 times in a random order for each time step from February-August 2017. When 15-point values per position were available, the standard deviation (referred to as STD) was calculated for each offset and each frequency. These values were then analyzed with respect to their population in order to determine the 99th quantile corresponding to the picking error for the considered time step. The overall uncertainty about the difference between the data of two-time steps was then defined as the sum of the errors calculated at each. The obtained values are listed in Table A1.

\begin{tabular}{|c|c|c|c|c|}
\hline \multicolumn{5}{|c|}{$\begin{array}{l}\text { Table A1 } \\
\text { Estimated Picking Errors } \\
\text { February to August } 2017\end{array}$} \\
\hline & \multicolumn{2}{|c|}{$P$ wave first arrival time } & \multicolumn{2}{|c|}{ Surface-wave phase velocity } \\
\hline & $\mathrm{STD}(\mathrm{ms})$ & No. of values & $\operatorname{STD}(\mathrm{m} / \mathrm{s})$ & No. of values \\
\hline February & 0.56 & 345 & 7.1 & 197 \\
\hline April & 0.59 & 373 & 4.3 & 734 \\
\hline June & 0.63 & 442 & 2.3 & 515 \\
\hline August & 0.73 & 435 & 5.3 & 167 \\
\hline
\end{tabular}

The point-by-point differences in arrival times represented for each source-geophone pair between each acquisition was then calculated, taking estimated errors into account, as presented in Figure A5. These diagrams allow for a first qualitative interpretation of the apparent subsurface $P$ wave velocity variations distributions (Dangeard et al., 2018). The diagonal corresponds to the arrival times at zero offset. The data represented below the diagonal are influenced by the structures encountered upstream of the geophone whereas above the diagonal they are influenced by the structures located downstream. The higher the source-receiver distance, the more the picked arrival time is affected by heterogeneities at depth. Time variations are negative between April and February on both banks. Between June and April, the differences are positive and included in the uncertainty. Between August and June, arrival time variations are positive. On the right bank, a specific zone is highlighted with differences included in the uncertainty or very high differences, for geophones and shots located between 35 and $40 \mathrm{~m}$. A poor coupling of geophones and sources at this location can explain these differences because they are systematic. In June, a rainy event occurred during the acquisition of the right bank. The soil saturation conditions were therefore not the same as the left banks. In addition, it was impossible to acquire seismic data along the whole profile and some seismograms were corrupted and were not fully exploited at the very end of the profile. This only affected travel times as presented in more details in Dangeard (2019). 
a. Apr. - Feb. / uncert. $1.15 \mathrm{~ms}$
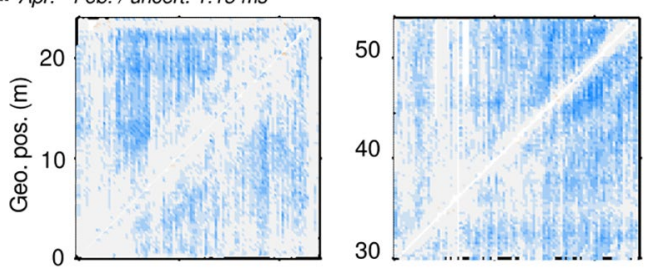

b. Jun. - Apr. / uncert. $1.22 \mathrm{~ms}$
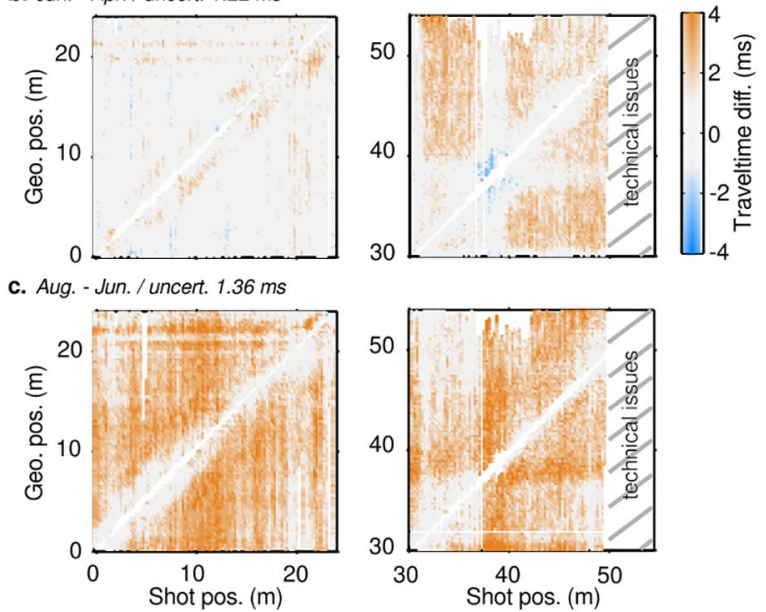

Figure A5. Absolute differences calculated between the picked arrival times of April and February (a, b), June and April (c, d) and August and June (e,f) 2017. The differences are represented according to the position of shots and geophones. These diagrams allow for a first qualitative interpretation of the apparent subsurface $P$ wave velocity variations distributions. The diagonal corresponds to the arrival times differences at zero offset. The data represented below the diagonal are influenced by the structures encountered upstream of the geophone whereas above the diagonal they are influenced by the structures located downstream. The color scale the picking uncertainty takes into account (in gray), determined by the statistical study. Due to a storm event in June, the last seismic shots of the acquisition on the right bank have been perturbed.

The point-by-point differences in the dispersion curves are given in the same way in Figure A6. Picked dispersion curves differences are juxtaposed for each extraction position along the line and represented as phase velocity differences pseudo-sections as a function of frequency. Changes are mainly positive between April and February and between June and April on both banks. Between August and June, differences in phase velocities are negative. There however is an interesting opposite behavior between low and high frequency dispersion data. High-frequency surface waves mainly investigate the shallowest layers when low-frequency surface waves correspond to deeper layers. The dispersion data thus do not respond to the saturation as globally as travel times. But this is only a rule of thumb and even if empirical relationships exist, inversion is preferred in this study. Seismic data variations in time are summarized in Table A2. 


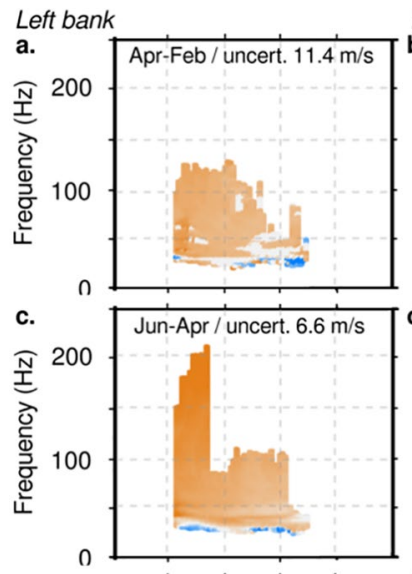

Right bank
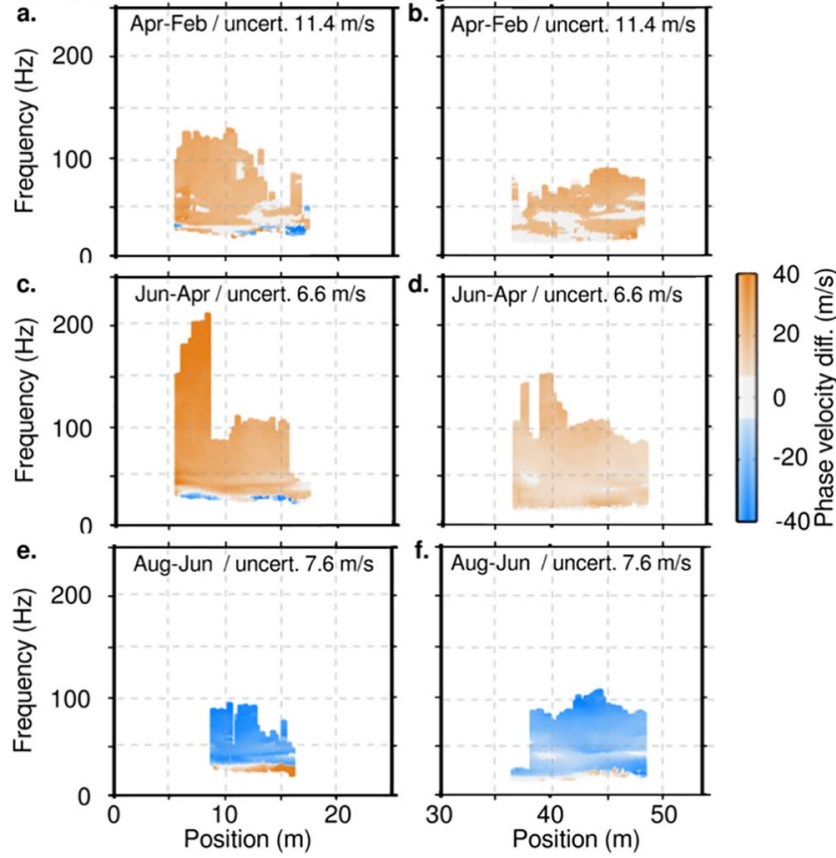

Figure A6. Absolute differences calculated between surface-wave dispersion curves of April-February (a, b), June and April (c, d), and August and June (e, f) 2017. Picked dispersion curves differences are juxtaposed for each extraction position along the line and phase velocity differences are plotted as a function of the window position and the frequency. The color scale considers the picking uncertainty (in gray), as determined by the statistical study (Table A1).

Table A2

Summary of the Global Variations Observed in the P Wave Arrival Times and the Surface-Wave Phase Velocities Along Both Banks (- Decrease; + Increase; = No Significant Variations)

\begin{tabular}{|c|c|c|c|c|c|c|}
\hline \multirow[b]{2}{*}{ Differences } & \multicolumn{2}{|c|}{ April-February } & \multicolumn{2}{|c|}{ June-April } & \multicolumn{2}{|c|}{ August-June } \\
\hline & LB & $\mathrm{RB}$ & LB & $\mathrm{RB}$ & LB & $\mathrm{RB}$ \\
\hline First arrival time & - & - & $=$ & + & + & + \\
\hline Phase velocity (surface) & + & + & + & + & - & - \\
\hline Phase velocity (depth) & - & $=$ & - & $=$ & + & + \\
\hline
\end{tabular}

\section{Appendix A5. Interpreting Seismic Data Variations in Time}

The apparent velocities described by picked first arrival time on a seismogram depend on VP which is sensitive to water saturation. VP in partially saturated non-consolidated media is lower than VP at the dry state. This is related to the increase in density due to partial saturation (in the pores, water replaces the air). From a water saturation threshold of about 90\%-95\% (depending on the materials), VP increases substantially until the full saturation is reached (the medium is harder to compress, see Bachrach and Nur (1998) for more details for instance). In such media, shear waves are very sensitive to small changes in water saturation when it is close to $0 \%$. VS tends to increase due to the creation of capillary bridges between grains rising their resistance to shear stress. Then, when air is replaced by water in the porous medium, VS tends to decrease (due to an increase in density, according to Cho \& Santamarina, 2001, for instance). As the surface waves observed on seismograms of vertical particle velocities are strongly dependent on VS, their phase velocities tend to be similarly influenced.

In order to use these qualitative relationships, frequency effects on elastic properties have to be considered. At low frequency, in a drained regime, the fluid pressure is unaffected by the seismic waves. On the contrary, at higher frequency, in an undrained regime, the fluid pressure changes when the wave passes through (Adelinet et al., 2018). According to Cleary (1978), the cut-off frequency $\left(f_{c}\right)$ between the drained and the undrained 
states is given by: $f_{c}=4 x k x K_{d} / \eta x L^{2}$, with $k$ an estimation of the average intrinsic permeability of the porous media $\left(10^{-12} \mathrm{~m}^{2}\right), K_{\mathrm{d}}$ the drained bulk modulus $\left(10^{9} \mathrm{~Pa}\right), \eta$ the water dynamic viscosity $\left(10^{-3} \mathrm{~Pa} . \mathrm{s}\right)$ and $L$ the flow length $(1.5 \mathrm{~m})$. A cut-off frequency of $1.8 \mathrm{~Hz}$ is obtained here which is lower than the source central frequency $(25-50 \mathrm{~Hz}$, depending on which type of wave is considered). We thus assume an undrained regime.

\section{Appendix A6. Example of Inverted Seismic Section in February 2017}

$P$ wave first arrival times were inverted for VP 2D sections in the framework of refraction tomography (Bauer et al., 2003; Schuster \& Quintus-Bosz, 1993; Sheehan et al., 2005). Obtained results (with the RAYFRACTC software, Rohdewald, 2011) are presented in Figure A7. Inversion is initialized using a smooth gradient initial model which is the 2D extension of the mean 1D model obtained directly from picked travel times, assuming velocity gradients in a 1D tabular medium (Gibson et al., 1979). The inversion process is stopped when velocity updates, global RMS errors, and maximum normalized residual do not vary (Figures A7c-A7f). From these criteria, 10 iterations appear needed (see details in Dangeard, 2019) to reach a plateau showing global RMS errors lower than $1 \mathrm{~ms}$ (Figures A7c and A7d). Ray coverage is presented in Figure A7b in order to control the domain actually investigated by $P$ waves. Body waves are mainly influenced by property variations along their path imposed by the velocity gradient with depth. This path senses the medium's properties between the source down to a maximum depth (which depends on the offset in the absence of strong contrasts) and then, up to the receiver. The longer the offset, the greater the depth of investigation. The drawback of the method here is clearly the lack of ray coverage at the subsurface. A

a. Left Bank - ESE

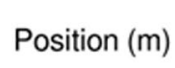

Right Bank WNW
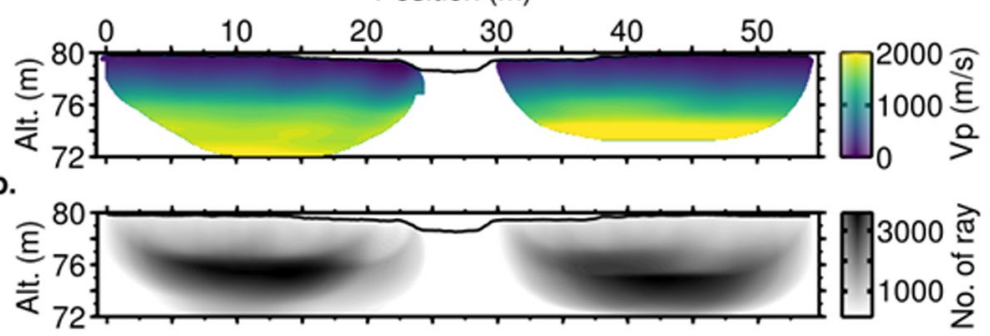

c. Left bank

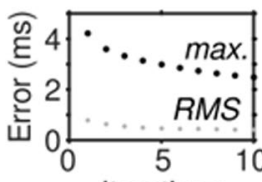

Iterations

\section{g. Left bank}

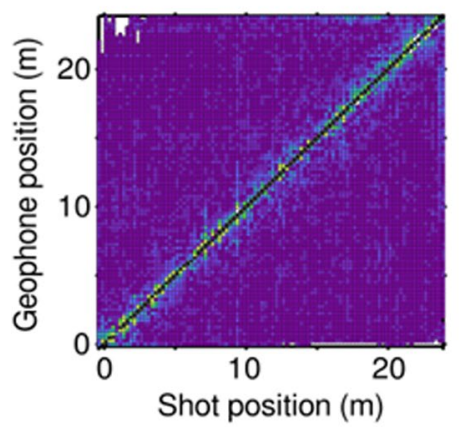

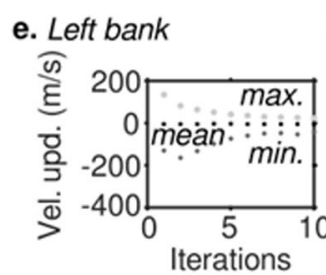

f. Right bank

\section{h. Right bank}

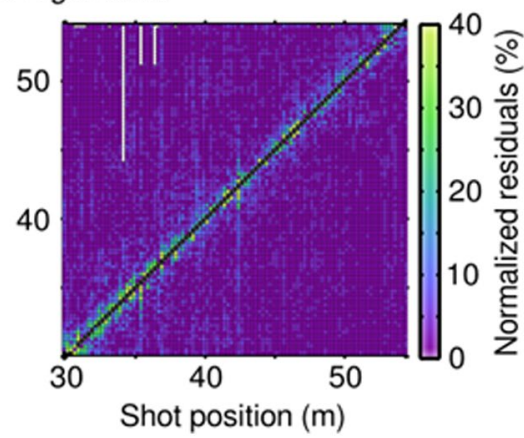

Figure A7. Detailed refraction tomography results for February 2017: (a) VP model obtained after a total of 10 iterations. (b) Ray coverage after 10 iterations. (c, d) RMS (in gray) and maximal absolute error (in black) variations through the iterations, for the left bank (c) and the right bank (d). (e, f) Minimum (dark gray), maximum (light gray), and mean(black) velocity update variations through iterations for the left bank (e) and the right bank (f). 
natural consequence is, in addition, the lateral homogeneity of the results that are mainly constrained by the gradient due to the WT.

Dispersion data were then inverted at each Xmid position for a 1D VS profile with depth, using the neighborhood algorithm developed by Sambridge (1999a, 1999b) and implemented by Wathelet (2008) and Wathelet et al. (2004) (see Pasquet \& Bodet, 2017, for near-surface applications along linear sections). Assuming a vertically heterogeneous layered model, theoretical dispersion curves are computed from the elastic parameters using Thomson-Haskel matrix propagator technique. The algorithm performs a stochastic search of the parameter space, namely VP, VS, density, and thickness of each layer. The parameterization of the model was first achieved without much prior information using a stack of 5 layers over a half-space. We imposed a maximum half-space depth of $0.625-10 \mathrm{~m}$ (based on the maximum recorded wavelength). VS and thickness $\mathrm{H}$ in each layer were free to evolve as follows: $10<\mathrm{VS}<1,250 \mathrm{~m} / \mathrm{s}$ and $0.125<H<2 \mathrm{~m}$ (VP and $\rho$, of lower influences on dispersion, are also model parameters but are not considered here).

Frequency-phase velocity curves with associated uncertainties, were inverted with 5 distinct and independent runs, generating a total of 50,500 models (100 iterations per run, $n_{\mathrm{s} 0}=100, n_{\mathrm{s}}=100$ and $n_{\mathrm{r}}=100$ ). No lateral constraints were applied during the inversions since we assumed surface-wave dispersion stacking to "naturally" smooth the data. Final sampled VS profiles with lowest misfits were eventually extracted, re-sampled in depth down to the max half-space depth, in order to estimate an average velocity structure (see Pasquet \& Bodet, 2017 for details about the workflow). Each 1D profile is then represented at its corresponding position so as to obtain the pseudo-2D VS section presented in Figure A8. VS variations due to the uncertainties of the inversion method at depth (related to poor resolution at great wavelength) were reinforced here by the absence of strong prior information. For each Xmid position, the maximum depth of investigation (DOI) is reached when the standard deviation between each $1 \mathrm{D}$ model is $150 \mathrm{~m} / \mathrm{s}$, we consider that the posterior error is too high (Figure A9).

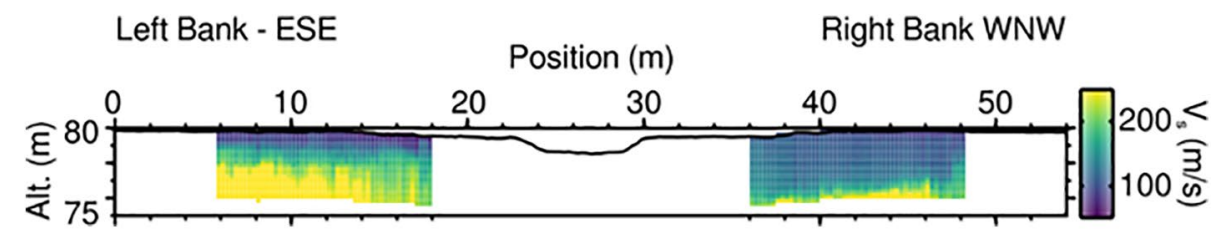

Figure A8. Surface-wave dispersion inversion results for February 2017: 2D VS sections.

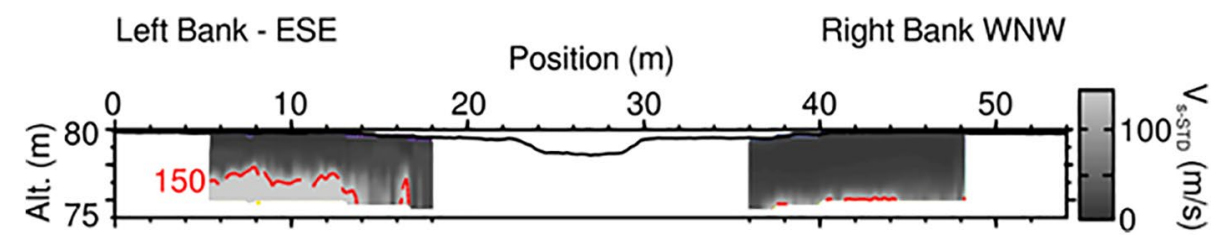

Figure A9. Surface-wave dispersion inversion results for February 2017: 2D sections of 1D results' standard deviation in VS (STD) obtained for inversions at each Xmid position along the line. The red limit marks the depth below which standard deviations are greater than $150 \mathrm{~m} / \mathrm{s}$.

Figure A7 clearly shows the natural smooth character of the VP model on one side and Figures A8 and A9 the lack of DOI of surface-wave inversion on the other side. VP appears dominated by the influence of water content (when saturated, different lithologies depict similar $P$ wave velocity value, at least with results a posteriori uncertainties). The VS model shows a better lateral resolution but involved materials, even if different in lithology, might have very close velocities that posterior uncertainties do not allow discriminating. This is why the combination of the ERT image with auger soundings, logs and penetrometer tests, was preferred to build the hydrofacieses model. 


\section{Data Availability Statement}

Surface-wave dispersion picking and inversions were performed thanks to the open-source packages SWIP (https://doi.org/10.3997/2214-4609.201602087), Seismic Unix (http://www.cwp.mines.edu/cwpcodes/) and Geopsy (http://www.geopsy.org/download.php). Numerical simulations were partly performed at the HPCaVe center, Sorbonne Université using the Ginette modeling code (https://doi.org/10.5281/ zenodo.4058821).

\section{References}

Acknowledgments

The authors kindly thank L. Longuevergne and J.-M. Mouchel for their constructive comments and recommendations. This work was supported by Sorbonne Université, the PIREN-Seine program, the ANR Project CRITEX ANR-11-EQPX-0011 in the framework of OZCAR network.
Aarts, E. H. L., \& Van Laarhoven, P. J. M. (1985). Statistical cooling: A general approach to combinatorial optimization problems. Philips Journal of Research, 40(4), 193-226.

Adelinet, M., Domínguez, C., Fortin, J., \& Violette, S. (2018). Seismic-refraction field experiments on Galapagos Islands: A quantitative tool for hydrogeology. Journal of Applied Geophysics, 148, 139-151. https://doi.org/10.1016/j.jappgeo.2017.10.009

Ajo-Franklin, J., Dou, S., Lindsey, N., Daley, T. M., Freifeld, B., Martin, E. R., et al. (2017). Time-lapse surface wave monitoring of permafrost thaw using distributed acoustic sensing and a permanent automated seismic source. In SEG Technical Program Expanded Abstracts 2017 (pp. 5223-5227). https://doi.org/10.1190/segam2017-17774027.1

Arts, R., Eiken, O., Chadwick, A., Zweigel, P., van der Meer, L., \& Zinszner, B. (2004). Monitoring of $\mathrm{CO}_{2}$ injected at Sleipner using timelapse seismic data. Energy, 29(9), 1383-1392. https://doi.org/10.1016/j.energy.2004.0310.1016/j.energy.2004.03.072

Bachrach, R., \& Nur, A. (1998). High-resolution shallow-seismic experiments in sand, Part I: Water table, fluid flow, and saturation. Geophysics, 63(4), 1225-1233. https://doi.org/10.1190/1.1444423

Bardini, L., Boano, F., Cardenas, M. B., Sawyer, A. H., Revelli, R., \& Ridolfi, L. (2013). Small-scale permeability heterogeneity has negligible effects on nutrient cycling in streambeds. Geophysical Research Letters, 40, 1118-1122. https://doi.org/10.1002/grl.50224

Batkova, K., Matula, S., Kechavarzi, C., \& Dresser, M. (2020). Differences in topsoil properties of a sandy loam soil under different tillage treatments. Paper presented at 9th World Congress of Soil Science, Soil Solutions for a Changing World, August 1-6, 2010a.

Bauer, K., Moeck, I., Norden, B., Schulze, A., Weber, M., \& Wirth, H. (2010). Tomographic $P$ wave velocity and vertical velocity gradient structure across the geothermal site Groß Schönebeck (NE German Basin): Relationship to lithology, salt tectonics, and thermal regime. Journal of Geophysical Research: Solid Earth, 115(B8), B08312. https://doi.org/10.1029/2009JB006895

Bauer, K., Schulze, A., Ryberg, T., Sobolev, S. V., \& Weber, M. H. (2003). Classification of lithology from seismic tomography: A case study from the Messum igneous complex, Namibia. Journal of Geophysical Research: Solid Earth, 108(B3), 2152-2167. https://doi. org/10.1016/j.geothermics.2010.12.00810.1029/2001jb001073

Benz-Navarrete, M. A. (2009). Mesures dynamiques lors du battage du pénétromètre PANDA 2 (Doctoral dissertation). Université Blaise Pascal—Clermont II. Retrieved from https://tel.archives-ouvertes.fr/tel-00725564

Bergamo, P., Dashwood, B., Uhlemann, S., Swift, R., Chambers, J. E., Gunn, D. A., \& Donohue, S. (2016a). Time-lapse monitoring of climate effects on earthworks using surface waves. Geophysics, 81(2), EN1-EN15. https://doi.org/10.1190/GEO2015-0275.1

Bergamo, P., Dashwood, B., Uhlemann, S., Swift, R., Chambers, J. E., Gunn, D. A., \& Donohue, S. (2016b). Time-lapse monitoring of fluid-induced geophysical property variations within an unstable earthwork using $P$ wave refraction. Geophysics, 81(4), EN17-EN27. https://doi.org/10.1190/geo2015-0276.1

Bergamo, P., \& Socco, L. V. (2016). $P$ and $S$ wave velocity models of shallow dry sand formations from surface wave multimodal inversion. Geophysics, 81(4), R197-R209. https://doi.org/10.1190/geo2015-0542.1

Bernard-Jannin, L., Sun, X., Teissier, S., Sauvage, S., \& Sánchez-Pérez, J.-M. (2017). Spatio-temporal analysis of factors controlling nitrate dynamics and potential denitrification hot spots and hot moments in groundwater of an alluvial floodplain. Ecological Engineering, 103, 372-384. https://doi.org/10.1016/j.ecoleng.2015.12.031

Berrhouma, A. (2018). Fonctionnement hydrothermique de l'interface nappe-rivière du bassin des Avenelles (Doctoral dissertation). Ecole Nationale Supérieure des Mines. Retrieved from http://www.theses.fr/2018PSLEM034.Location

Beven, K. (2006). A manifesto for the equifinality thesis. Journal of Hydrology, 320, 18-36. https://doi.org/10.1016/j.jhydrol.2005.07.007

Binley, A., Cassiani, G., \& Deiana, R. (2010). Hydrogeophysics: Opportunities and challenges. Bolletino di Geofisica Teorica et Applicata, 51(4), 267-284.

Binley, A., Hubbard, S. S., Huisman, J. A., Revil, A., Robinson, D. A., Singha, K., \& Slater, L. D. (2015). The emergence of hydrogeophysics for improved understanding of subsurface processes over multiple scales. Water Resources Research, 51(6), 3837-3866. https://doi. org/10.1002/2015WR017016

Biot, M. A. (1956a). Theory of propagation of elastic waves in a fluid-saturated porous solid. I. Low-frequency range. Journal of the Acoustical Society of America, 28(2), 168-178. https://doi.org/10.1121/1.1908239

Biot, M. A. (1956b). Theory of propagation of elastic waves in a fluid-saturated porous solid. II. Higher frequency range. Journal of the Acoustical Society of America, 28(2), 179-191. https://doi.org/10.1121/1.1908241

Blazevic, L. A., Bodet, L., Pasquet, S., Linde, N., Jougnot, D., \& Longuevergne, L. (2020). Time-lapse seismic and electrical monitoring of the vadose zone during a controlled infiltration experiment at the ploemeur hydrological observatory, France. Water, 12(5), 1230. https://doi.org/10.3390/w12051230

Boano, F., Demaria, A., Revelli, R., \& Ridolfi, L. (2010). Biogeochemical zonation due to intrameander hyporheic flow. Water Resources Research, 46(2), 1-13. https://doi.org/10.1029/2008WR007583

Bodet, L., Abraham, O., \& Clorennec, D. (2009). Near-offsets effects on Rayleigh-wave dispersion measurements: Physical modeling. Journal of Applied Geophysics, 68(1), 95-103. https://doi.org/10.1016/j.jappgeo.2009.02.012

Bodet, L., van Wijk, K., Bitri, A., Abraham, O., Côte, P., Grandjean, G., \& Leparoux, D. (2005). Surface-wave inversion limitations from laser-Doppler physical modeling. Journal of Environmental and Engineering Geophysics, 10(2), 151-162. https://doi.org/10.2113/ JEEG10.2.151

Brunner, P., Cook Peter, G., \& Simmons, C. T. (2011). Disconnected surface water and groundwater: From theory to practice. Ground Water, 49(4), 460-467. https://doi.org/10.1111/j.1745-6584.2010.00752.x

Brunner, P., Therrien, R., Renard, P., Simmons, C. T., \& Franssen, H. J. H. (2017). Advances in understanding river-groundwater interactions. Reviews of Geophysics, 55(3), 818-854. https://doi.org/10.1002/2017RG000556 
Cameron, A., \& Knapp, C. C. (2009). Geostatistical analysis of near-surface geophysical data : Results from the P-Reactor area at Savannah River Site, South Carolina. In AGU Fall Meeting Abstracts (Vol. 2009, pp. H53B-0916).

Cardenas, M. B. (2009). Stream-aquifer interactions and hyporheic exchange in gaining and losing sinuous streams. Water Resources Research, 45(6), 1-13. https://doi.org/10.1029/2008WR007651

Cardenas, M. B., Wilson, J. L., \& Zlotnik, V. A. (2004). Impact of heterogeneity, bed forms, and stream curvature on subchannel hyporheic exchange. Water Resources Research, 40(8), W08307. https://doi.org/10.1029/2004WR003008

Chadwick, A., Williams, G., Delepine, N., Clochard, V., Labat, K., Sturton, S., et al. (2010). Quantitative analysis of time-lapse seismic monitoring data at the Sleipner $\mathrm{CO}_{2}$ storage operation. The Leading Edge, 29(2), 170-177. https://doi.org/10.1190/1.3304820

Cho, G., \& Santamarina, J. (2001). Unsaturated particulate materials-Particle-level studies. Journal of Geotechnical and Geoenvironmental Engineering, 127(1), 84-96. https://doi.org/10.1061/(ASCE)1090-024110.1061/(asce)1090-0241(2001)127:1(84)

Chorover, J., Kretzschmar, R., Garcia-Pichel, F., \& Sparks, D. L. (2007). Soil biogeochemical processes within the critical zone. Elements, 3(5), 321-326. https://doi.org/10.2113/gselements.3.5.321

Cleary, M. P. (1978). Elastic and dynamic response regimes of fluid-impregnated solids with diverse microstructures. International Journal of Solids and Structures, 14(10), 795-819. https://doi.org/10.1016/0020-7683(78)90072-0

Conant, B. (2004). Delineating and quatifying groundwater discharge zones using streambed temperatures. Ground Water, 42(2), $243-257$. https://doi.org/10.1111/j.1745-6584.2004.tb02671.x

Conant, B., Robinson, C. E., Hinton, M. J., \& Russel, H. A. J. (2019). A framework for conceptualizing groundwater-surface water interactions and identifying potential impacts on water quality, water quantity, and ecosystems. Journal of Hydrology, 574, 609-627. https:// doi.org/10.1016/j.jhydrol.2019.04.050

Coscia, I., Greenhalgh, S. A., Linde, N., Doetsch, J., Marescot, L., Günther, T., et al. (2011). 3D crosshole ERT for aquifer characterization and monitoring of infiltrating river water. Geophysics, 76(2), G49-G59. https://doi.org/10.1190/1.3553003

Cucchi, K., Rivière, A., Baudin, A., Berrhouma, A., Durand, V., Rejiba, F. \& Flipo, N. (2018). LOMOS-mini: A coupled system quantifying transient water and heat exchanges in streambeds. Journal of Hydrology, 561, 1037, 1047, https://doi.org/10.1016/j.jhydrol.2017.10.074

Cucchi, K., Rivière, A., Rubin, Y., \& Flipo, N. (2019). Estimation des échanges nappe-rivière locaux et de leur variation le long d'un réseau hydrographique: Développement d'un capteur innovant et application au bassin agricole des Avenelles. Mesures hydrogéophysiques et biogéochimiques haute-fréquence et "time-lapse" des interfaces nappe-rivière : évolution des ressources en eau.

Dangeard, M. (2019). Développement d'une approche "time-lapse” des méthodes sismiques pour l'hydrogéophysique et la compréhension de la dynamique des hydrosystèmes (Doctoral dissertation). Sorbonne Université. Retrieved from https://www.theses.fr/2019SORUS476

Dangeard, M., Bodet, L., Pasquet, S., Thiesson, J., Guérin, R., Jougnot, D., \& Longuevergne, L. (2018). Estimating picking errors in near-surface seismic data to enable their time-lapse interpretation of hydrosystems. Near Surface Geophysics, 16(6), 613-625. https:// doi.org/10.1002/nsg.12019

de Marsily, G., Delay, F., Gonçalvès, J., Renard, P., Teles, V., \& Violette, S. (2005). Dealing with spatial heterogeneity. Hydrogeology Journal, 13(1), 161-183. https://doi.org/10.1007/s10040-004-0432-3

Descloîtres, M., Ribolzi, O., Le Troquer, Y., \& Thiebaux, J. P. (2003). Study of infiltration in a Sahelian gully erosion area using time-lapse resistivity mapping. Journal of Applied Geophysics, 53(3), 229-253. https://doi.org/10.1016/S0341-8162(03)00038-9

Doetsch, J., Linde, N., Pessognelli, M., Green, A. G., \& Günther, T. (2012). Constraining 3D electrical resistance tomography with GPR reflection data for improved aquifer characterization. Journal of Applied Geophysics, 78, 68-76. https://doi.org/10.1016/j.jappgeo.2011.04.008

Dudley-Southern, M., \& Binley, A. (2015). Temporal responses of groundwater-surface water exchange to successive storm events. Water Resources Research, 51(2), 1112-1126. https://doi.org/10.1002/2014WR016623

Fan, Y., Grant, G., \& Anderson, S. P. (2019). Water within, moving through, and shaping the Earth's surface: Introducing a special issue on water in the critical zone. Hydrological Processes, 33(25), 3146-3151. https://doi.org/10.1002/hyp.13638

Floury, P., Gaillardet, J., Tallec, G., Ansart, P., Bouchez, J., Louvat, P., \& Gorge, C. (2018). Chemical weathering and $\mathrm{CO}_{2}$ consumption rate in a multilayered-aquifer dominated watershed under intensive farming: The Orgeval Critical Zone Observatory, France. Hydrological Processes, 33(2), 195-213. https://doi.org/10.1002/hyp.13340

Fox, A., Laube, G., Schmidt, C., Fleckenstein, J. H., \& Arnon, S. (2016). The effect of losing and gaining flow conditions on hyporheic exchange in heterogeneous streambeds: Hyporheic exchange in heterogeneous streambeds. Water Resources Research, 52, $7460-7477$. https://doi.org/10.1002/2016WR018677

Freeze, R. A., \& Witherspoon, P. A. (1967). Theoretical analysis of regional groundwater flow: 2. Effect of water-table configuration and subsurface permeability variation. Water Resources Research, 3(2), 623-634. https://doi.org/10.1029/WR003i002p00623

Gaillardet, J., Braud, I., Hankard, F., Anquetin, S., Bour, O., Dorfliger, N., et al. (2018). OZCAR : The French network of critical zone observatories. Vadose Zone Journal, 17(1), 1-24. https://doi.org/10.2136/vzj2018.04.0067

Gallois, N., Rivière, A., Flipo, N., Schuite, J., Cucchi, K., Kiliç, D., et al. (2019). Stratégie de calibration et modélisation de l'hydrosystème du bassin versant des Avenelles. PIREN Seine. Rapports de synthèse de fin de phase VII du programme PIREN Seine, 4, pp. 47-72, Mesures hydrogéophysiques et biogéochimiques haute-fréquence et "time-lapse" des interfaces nappe-rivière : évolution des ressources en eau.

Garnier, J., Anglade, J., Benoit, M., Billen, G., Puechc, T., Ramarson, A., et al. (2016). Reconnecting crop and cattle farming to reduce nitrogen losses to river water of an intensive agricultural catchment (Seine basin, France): Past, present, and future. Environmental Science \& Policy, 63, 76-90. https://doi.org/10.1016/j.envsci.2016.04.019

Genereux, D., Leahy, S., Mitasova, H., Kennedy, C., \& Corbett, D. (2008). Spatial and temporal variability of streambed hydraulic conductivity in West Bear Creek, North Carolina, USA. Journal of Hydrology, 358, 332-353. https://doi.org/10.1016/j.jhydrol.2008.06.017

Gibson, B. S., Odegard, M. E., \& Sutton, G. H. (1979). Nonlinear least-squares inversion of travel time data for a linear velocity-depth relationship. Geophysics, 44(2), 185-194. https://doi.org/10.1190/1.1440960

Goderniaux, P., Davy, P., Bresciani, E., de Dreuzy, J. R., \& Le Borgne, T. (2013). Partitioning a regional groundwater flow system into shallow local and deep regional flow compartments. Water Resources Research, 49(4), 2274-2286. https://doi.org/10.1002/wrcr.20186

Gomez-Velez, J. D., Krause, S., \& Wilson, J. L. (2014). Effect of low-permeability layers on spatial patterns of hyporheic exchange and groundwater upwelling. Water Resources Research, 50, 5196-5215. https://doi.org/10.1002/2013WR015054

Grelle, G., \& Guadagno, F. M. (2009). Seismic refraction methodology for groundwater level determination: Seismic index. Journal of Applied Geophysics, 68(3), 301-320. https://doi.org/10.1016/j.jappgeo.2009.02.001

Griebler, C., \& Avramov, M. (2015). Groundwater ecosystem services: A review. Freshwater Science, 34(1), 355-367. https://doi. $\operatorname{org} / 10.1086 / 679903$

Harvey, J., \& Gooseff, M. (2015). River corridor science: Hydrologic exchange and ecological consequences from bedforms to basins. Water Resources Research, 51(9), 6893-6922. https://doi.org/10.1002/2015WR017617 
Hester, E. T., Eastes, L. A., \& Widdowson, M. A. (2019). Effect of surface water stage fluctuation on mixing-dependent hyporheic denitrification in riverbed dunes. Water Resources Research, 55, 4668-4687. https://doi.org/10.1029/2018WR024198

Hlavek, R., \& Dubreuil, P. (1965). Recherches hydrologiques sur bassins représentatifs au ministère de l'agriculture: Le secteur pilote de l'Orgeval objectifs et méthodes d'exploitation.

Hubbard, S. S., \& Linde, N. (2011). Hydrogeophysics. Treatise on water science (pp. 401-434). Elsevier. https://doi.org/10.1016/ b978-0-444-53199-5.00043-9

Ikeda, T., Tsuji, T., Takanashi, M., Kurosawa, I., Nakatsukasa, M., Kato, A., et al. (2017). Temporal variation of the shallow subsurface at the Aquistore $\mathrm{CO}_{2}$ storage site associated with environmental influences using a continuous and controlled seismic source. Journal of Geophysical Research: Solid Earth, 122(4), 2859-2872. https://doi.org/10.1002/2016JB013691

Irvine, D. J., Brunner, P., Hendricks Franssen, H.-J., \& Simmons, G. T. (2012). Heterogeneous or homogeneous? Implications of simplifying heterogeneous streambeds in models of losing streams. Journal of Hydrology, 424-425, 16-23. https://doi.org/10.1016/j. jhydrol.2011.11.051

Jougnot, D., Linde, N., Haarder, E. B., \& Looms, M. C. (2015). Monitoring of saline tracer movement with vertically distributed self-potential measurements at the HOBE agricultural test site, Voulund, Denmark. Journal of Hydrology, 521, 314-327. https://doi.org/10.1016/j. jhydrol.2014.11.041

Kalbus, E., Reinstorf, F., \& Schirmer, M. (2006). Measuring methods for groundwater-surface water interactions : A review. Hydrology and Earth System Sciences, 10, 873-887. https://doi.org/10.5194/hess-10-873-2006

Kalbus, E., Schmidt, C., Molson, J. W., Reinstorf, F., \& Schirmer, M. (2009). Influence of aquifer and streambed heterogeneity on the distribution of groundwater discharge. Hydrology and Earth System Sciences, 13(1), 69-77. https://doi.org/10.5194/hess-13-69-2009

Kennedy, C. D., Genereux, D. P., Corbett, D. R., \& Mitasova, H. (2009a). Relationships among groundwater age, denitrification, and the coupled groundwater and nitrogen fluxes through a streambed. Water Resources Research, 45, W09402. https://doi. org/10.1029/2008WR007400R007400

Kennedy, C. D., Genereux, D. P., Corbett, D. R., \& Mitasova, H. (2009b). Spatial and temporal dynamics of coupled groundwater and nitrogen fluxes through a streambed in an agricultural watershed. Water Resources Research, 45, W09401. https://doi.org/10.1029/2008wr007397

Konstantaki, L. A., Carpentier, S., Garofalo, F., Bergamo, P., \& Socco, L. V. (2013). Determining hydrological and soil mechanical parameters from multichannel surface-wave analysis across the Alpine fault at Inchbonnie, New Zealand. Near Surface Geophysics, 11(4), 435-448. https://doi.org/10.3997/1873-0604.2013019

Krause, S., Blume, T., \& Cassidy, N. J. (2012). Investigating patterns and controls of groundwater up-welling in a lowland river by combining fiber-optic distributed temperature sensing with observations of vertical hydraulic gradients. Hydrology and Earth System Sciences, 16(6), 1775-1792. https://doi.org/10.5194/hess-16-1775-2012

Krause, S., Boano, F., Cuthbert, M. O., Fleckenstein, J. H., \& Lewandowski, J. (2014). Understanding process dynamics at aquifer-surface water interfaces: An introduction to the special section on new modeling approaches and novel experimental technologies. Water Resources Research, 50(2), 1847-1855. https://doi.org/10.1002/2013WR014755

Krause, S., Freer, J., Hannah, D. M., Howden, N. J., Wagener, T., \& Worrall, F. (2014). Catchment similarity concepts for understanding dynamic biogeochemical behavior of river basins. Hydrological Processes, 28(3), 1554-1560. https://doi.org/10.1002/hyp.10093

Krause, S., Hannah, D. M., Fleckenstein, J. H., Heppell, C. M., Kaeser, D., Pickup, R., et al. (2011). Inter-disciplinary perspectives on processes in the hyporheic zone. Ecohydrology, 4(4), 481-499. https://doi.org/10.1002/eco.176

Krause, S., Tecklenburg, C., Munz, M., \& Naden, E. (2013). Streambed nitrogen cycling beyond the hyporheic zone: Flow controls on horizontal patterns and depth distribution of nitrate and dissolved oxygen in the upwelling groundwater of a lowland river. Journal of Geophysical Research: Biogeosciences, 118(1), 54-67. https://doi.org/10.1029/2012JG002122

Laube, G., Schmidt, C., \& Fleckenstein, J. H. (2018). The systematic effect of streambed conductivity heterogeneity on hyporheic flux and residence time. Advances in Water Resources, 122, 60-69. https://doi.org/10.1016/j.advwatres.2018.10.003

Leek, R., Wu, J. Q., Wang, L., Hanrahan, T. P., Barber, M. E., Qiu, H., \& Qiu, H. (2009). Heterogeneous characteristics of streambed saturated hydraulic conductivity of the Touchet River, south eastern Washington, USA. Hydrological Processes, 23(8), 1236-1246. https:// doi.org/10.1002/hyp.7258

Liang, X., Quan, D., Jin, M., Liu, Y., \& Zhang, R. (2012). Numerical simulation of groundwater flow patterns using flux as upper boundary. Hydrological Processes, 27, 3475-3483. https://doi.org/10.1002/hyp.9477

Linde, N., Coscia, I., Doetsch, J., Greenhalgh, S., Vogt, T., Schneider, P., \& Green, A. (2010). Hydrogeophysical studies in unrestored and restored river corridors of the Thur River, Switzerland. First Break, 28(8), 69-75. https://doi.org/10.3997/1365-2397.28.8.40745

Linde, N., Doetsch, J., Jougnot, D., Genoni, O., Duerst, Y., Minsley, B. J., et al. (2011). Self-potential investigations of a gravel bar in a restored river corridor. Hydrology and Earth System Sciences, 15(3), 729-742. https://doi.org/10.5194/hess-15-729-2011

Loke, M. H., \& Barker, R. D. (1996). Rapid least-square inversion of apparent resistivity pseudosections using a quasi-Newton method. Geophysical Prospecting, 44(1), 131-152. https://doi.org/10.1111/j.1365-2478.1996.tb00142.x

Loumagne, C., \& Tallec, G. (2013). L'observation long terme en environnement: Exemple du bassin versant de l'Orgeval. QUAE.

Lu, N., \& Likos, W. J. (2004). Unsaturated soil mechanics. Wiley.

Lu, Z. (2014). Feasibility of using a seismic surface wave method to study seasonal and weather effects on shallow surface soils. Journal of Environmental and Engineering Geophysics, 19(2), 71-85. https://doi.org/10.2113/JEEG19.2.71

Maineult, A. (2016). Estimation of the electrical potential distribution along metallic casing from surface self-potential profile. Journal of Applied Geophysics, 129, 66-78. https://doi.org/10.1016/j.jappgeo.2016.03.038

Mari, J.-L., \& Porel, G. (2008). 3D seismic imaging of a near-surface heterogeneous aquifer: A case study. Oil and Gas Science and Technology - Revue IFP, 63(2), 179-201. https://doi.org/10.2516/ogst:2007077

Marion, A., Packman, A., Zaramella, M., \& Bottacin-Busolin, A. (2008). Hyporheic flows in stratified beds. Water Resources Research, 44, W09433. https://doi.org/10.1029/2007WR006079

Mariotti, A., \& Letolle, R. (1977). Application de l'étude isotopique de l'azote en hydrologie et en hydrogéologie-analyse des résultats obtenus sur un exemple précis: Le Bassin de Melarchez (Scine-et-Marne, France). Journal of Hydrology, 33(1-2), 157-172. https://doi. org/10.1016/0022-1694(77)90105-6

McLachlan, P. J., Chambers, J. E., Uhlemann, S. S., \& Binley, A. (2017). Geophysical characterization of the groundwater-surface water interface. Advances in Water Resources, 109, 302-319. https://doi.org/10.1016/j.advwatres.2017.09.016

Mégnien, C. (1973). Premiers résultats de l'étude géologique et structurale du réservoir aquifère de la nappe des calcaires de champigny en brie. Rapport BRGM.

Morel-Seytoux, J. H. (2009). The turning factor in the estimation of stream aquifer seepage. Ground Water, 47(2), 205-212. https://doi. org/10.1111/j.1745-6584.2008.00512.x 
Morris, D. A., \& Johnson, A. I. (1967). Summary of hydrologic and physical properties of rock and soil materials, as analyzed by the hydrologic laboratory of the U.S. Geological Survey, 1948-60. Water Supply Paper 1839-D, 1839, 42. https://doi.org/10.3133/wsp1839D

Mouchel, J.-M., Rocha, S., Rivière, A., \& Tallec, G. (2019). Caractérisation de la géochimie des interfaces nappe-rivière du bassin des Avenelles. https://doi.org/10.13140/RG.2.2.22898.32967

Mouhri, A., Flipo, N., Rejiba, F., de Fouquet, C., Bodet, L., Kurtulus, B., et al. (2013). Designing a multi-scale sampling system of stream-aquifer interfaces in a sedimentary basin. Journal of Hydrology, 504, 194-206. https://doi.org/10.1016/j.jhydrol.2013.09.036

National Research Council (NRC). (2001). Basic research opportunities in Earth science. The National Academies Press.

Neira, J. M. T., Tallec, G., Andréassian, V., \& Mouchel, J. M. (2020). A combined mixing model for high-frequency concentration-discharge relationships. Journal of Hydrology, 591, 125559. https://doi.org/10.1016/j.jhydrol.2020.125559

Oldham, C. E., Farrow, D. E., \& Peiffer, S. (2013). A generalized Damköhler number for classifying material processing in hydrological systems. Hydrology and Earth System Sciences, 17(3), 1133-1148. https://doi.org/10.5194/hess-17-1133-2013

O'Neill, A. (2003). Full-waveform reflectivity for modeling, inversion and appraisal of seismic surface wave dispersion in shallow site investigations (Doctoral dissertation). University of Western Australia. Retrieved from https://www.slideshare.net/AdamONeill6/oneill2003phd

O'Neill, A., Dentith, M., \& List, R. (2003). Full-waveform P-SV reflectivity inversion of surface waves for shallow engineering applications. Exploration Geophysics, 34(3), 158-173. https://doi.org/10.1071/EG03158

Packman, A. I., Marion, A., Zaramella, M., Chen, C., Gaillard, J.-F., \& Keane, D. T. (2006). Development of layered sediment structure and its effects on pore water transport and hyporheic exchange. Water, Air, \& Soil Pollution: Focus, 6, 433-442. https://doi.org/10.1007/ s11267-006-9057-y

Pardo-Alvarez, A., Fleckenstein, J., Schilling, O., Trauth, N., Hunkeler, D., \& Brunner, P. (2018). Closing the conceptual gap between the hyporheic zone and the river corridor. Conference: Computational Methods in Water Resources (CMWR)At: Saint Malo.

Park, C. B., Miller, R. D., \& Xia, J. (1999). Multichannel analysis of surface waves. Geophysics, 64(3), 800-808. https://doi. org/10.1190/1.1444590

Parsekian, A. D., Singha, K., Minsley, B. J., Holbrook, W. S., \& Slater, L. (2015). Multiscale geophysical imaging of the critical zone. Reviews of Geophysics, 53(1), 1-26. https://doi.org/10.1002/2014RG000465

Pasquet, S., \& Bodet, L. (2017). SWIP: An integrated workflow for surface-wave dispersion inversion and profiling. Geophysics, 82(6), WB47-WB61. https://doi.org/10.1190/geo2016-0625.1

Pasquet, S., Bodet, L., Bergamo, P., Guérin, R., Martin, R., Mourgues, R., \& Tournat, V. (2016). Small scale seismic monitoring of varying water level in granular media. Vadose Zone Journal, 15(7), 1-14. https://doi.org/10.2136/vzj2015.11.0142

Pasquet, S., Bodet, L., Dhemaied, A., Mouhri, A., Vitale, Q., Rejiba, F., et al. (2015). Detecting different water table levels in a shallow aquifer with combined P-, surface and SH-wave surveys: Insights from VP/VS or Poisson's ratios. Journal of Applied Geophysics, 13, 28-50. https://doi.org/10.1016/j.jappgeo.2014.12.005

Pasquet, S., Bodet, L., Longuevergne, L., Dhemaied, A., Camerlynck, C., Rejiba, F., \& Guérin, R. (2015). 2D characterization of near-surface : Surface-wave dispersion inversion versus refraction tomography. Near Surface Geophysics, 13(4), 315-332. https://doi. org/10.3997/1873-0604.2015028

Pasquet, S., Holbrook, W. S., Carr, B. J., \& Sims, K. W. W. (2016). Geophysical imaging of shallow degassing in a Yellowstone hydrothermal system. Geophysical Research Letters, 43(23), 12027-12035. https://doi.org/10.1002/2016GL071306

Pinay, G., Peiffer, S., Dreuzy, J. D., Krause, S., Hannah, D. M., Fleckenstein, J. H., et al. (2015). Upscaling nitrogen removal capacity from local hotspots to low stream orders' drainage basins. Ecosystems, 18, 1101-1120. https://doi.org/10.1007/s10021-015-9878-5

Pride, S. R. (2005). Relationships between seismic and hydrological properties. Hydrogeophysics, 50, 253-290. https://doi. org/10.1007/1-4020-3102-5_9

Pryshlak, T. T., Sawyer, A. H., Stonedahl, S. H., \& Soltanian, M. R. (2015). Multiscale hyporheic exchange through strongly heterogeneous sediments. Water Resources Research, 51(11), 9127-9140. https://doi.org/10.1002/2015WR017293

Ranalli, A. J., \& Macalady, D. L. (2010). The importance of the riparian zone and in-stream processes in nitrate attenuation in undisturbed and agricultural watersheds-A review of the scientific literature. Journal of Hydrology, 389, 406-415. https://doi.org/10.1016/j. jhydrol.2010.05.045

Rivière, A., Flipo, N., Goblet, P., Cucchi, K., Berrhouma, A., Scheifer, J., et al. (2019). Réseau de suivi "MOnitoring LOcal des échanges NAppe Rivière" du bassin de l'Orgeval: Fonctionnement hydrogéophysique des interfaces nappe-rivière et conséquences sur la ressource en eau. Mesures hydrogéophysiques et biogéochimiques haute fréquence et "time-lapse" des interfaces nappe-rivière : évolution des ressources en eau.

Rivière, A., Gonçalvès, J., Jost, A., \& Font, M. (2014). Experimental and numerical assessment of transient stream-aquifer exchange during disconnection. Journal of Hydrology, 517, 574-583. https://doi.org/10.1016/j.jhydrol.2014.05.040

Rivière, A., Jost, A., Gonçalvès, J., \& Font, M. (2019). Pore water pressure evolution below a freezing front under saturated conditions: Large-scale laboratory experiment and numerical investigation. Cold Regions Science and Technology, 158, 76-94. https://doi. org/10.1016/j.coldregions.2018.11.005

Rohdewald, S. R. (2011). Interpretation of first-arrival travel times with wave path eikonal travel time inversion and wavefront refraction method. In Symposium on the Application of Geophysics to Engineering and Environmental Problems. EEGS. https://doi. org/10.4133/1.3614086

Rosenberry, D., \& Pitlick, J. (2009). Local-scale spatial and temporal variability of seepage in a shallow gravel bed river. Hydrological Processes, 23(23), 3306-3318. https://doi.org/10.1002/hyp.7433

Rubin, Y., \& Hubbard, S. S. (2006). Hydrogeophysics (Vol. 50). Springer Science \& Business Media.

Sabor, K., Jougnot, D., Guerin, R., Steck, B., Henault, J.-M., Apffel, A., \& Vautrin, D. (2021). A data mining approach for improved interpretation of ERT inverted sections using the DBSCAN clustering algorithm. Geophysical Journal International, 225, 1304-1318. https:// doi.org/10.1093/gji/ggab023

Salehin, M., Packman, A. I., \& Paradis, M. (2004). Hyporheic exchange with heterogeneous streambeds: Laboratory experiments and modeling. Water Resources Research, 40(11), W11504. https://doi.org/10.1029/2003WR002567

Sambridge, M. (1999a). Geophysical inversion with a neighbourhood algorithm-I. Searching a parameter space. Geophysical Journal International, 138(2), 479-494. https://doi.org/10.1046/j.1365-246X.1999.00876.x

Sambridge, M. (1999b). Geophysical inversion with a neighborhood algorithm-II. Appraising the ensemble. Geophysical Journal International, 138(3), 727-746. https://doi.org/10.1046/j.1365-246x.1999.00900.x

Sanford, W. (2002). Recharge and groundwater models: An overview. Hydrogeology Journal, 10, 110-120. https://doi.org/10.1007/ s10040-001-0173-5 
Sawyer, A. H. (2015). Enhanced removal of groundwater-borne nitrate in heterogeneous aquatic sediments. Geophysical Research Letters, 42(2), 403-410. https://doi.org/10.1002/2014GL062234

Sawyer, A. H., \& Cardenas, M. B. (2009). Hyporheic flow and residence time distributions in heterogeneous cross-bedded sediment. Water Resources Research, 45(8), W08406. https://doi.org/10.1029/2008WR007632

Schilling, O. S., Cook, P. G., \& Brunner, P. (2019). Beyond classical observations in hydrogeology: The advantages of including exchange flux, temperature, tracer concentration, residence time, and soil moisture observations in groundwater model calibration. Reviews of Geophysics, 57(1), 146-182. https://doi.org/10.1029/2018RG000619

Schneider, P., Vogt, T., Schirmer, M., Doetsch, J., Linde, N., Pasquale, N., et al. (2011). Towards improved instrumentation for assessing river-groundwater interactions in a restored river corridor. Hydrology and Earth System Sciences, 15(8), 2531-2549. https://doi. org/10.5194/hess-15-2531-2011

Schuster, G. T., \& Quintus-Bosz, A. (1993). Wave path eikonal travel time inversion: Theory. Geophysics, 58(9), 1314-1323. https://doi. org/10.1190/1.1443514

Sheehan, J. R., Doll, W. E., \& Mandell, W. A. (2005). An evaluation of methods and available software for seismic refraction tomography analysis. Journal of Environmental and Engineering Geophysics, 10(1), 21-34. https://doi.org/10.2113/JEEG10.1.21

Shuai, P., Cardenas, M. B., Knappett, P. S. K., Bennett, P. C., \& Neilson, B. T. (2017). Denitrification in the banks of fluctuating rivers: The effects of river stage amplitude, sediment hydraulic conductivity and dispersivity, and ambient groundwater flow. Water Resources Research, 53(9), 7951-7967. https://doi.org/10.1002/2017WR020610

Singha, K., Pidlisecky, A., Day-Lewis, F. D., \& Gooseff, M. N. (2008). Electrical characterization of non-Fickian transport in groundwater and hyporheic systems. Water Resources Research, 46(4), W00D07. https://doi.org/10.1029/2008wr007048

Socco, L. V., \& Strobbia, C. (2004). Surface-wave method for near-surface characterization: A tutorial. Near-Surface Geophysics, 2(4), 165185. https://doi.org/10.3997/1873-0604.2004015

Song, X., Chen, X., Stegen, J., Hammond, G., Song, H. S., Dai, H., et al. (2018). Drought conditions maximize the impact of high-frequency flow variations on thermal regimes and biogeochemical function in the hyporheic zone. Water Resources Research, 54(10), 7361-7382. https://doi.org/10.1029/2018WR022586

St Clair, J., Moon, S., Holbrook, W. S., Perron, J. T., Riebe, C. S., Martel, S. J., et al. (2015). Geophysical imaging reveals topographic stress control of bedrock weathering. Science, 350(6260), 534-538. https://doi.org/10.1126/science.aab2210

Tallec, G., Ansart, P., Guérin, A., Delaigue, O., \& Blanchouin, A. (2015). Observatoire ORACLE [Data set]. Irstea. https://doi.org/10.17180/ obs.oracle

Tang, Q., Kurtz, W., Brunner, P., Vereecken, H., \& Franssen, H.-J. H. (2015). Characterization of river-aquifer exchange fluxes: The role of spatial patterns of riverbed hydraulic conductivities. Journal of Hydrology, 531, 111-123. https://doi.org/10.1016/j.jhydrol.2015.08.019

Trauth, N., \& Fleckenstein, J. H. (2017). Single discharge events increase reactive efficiency of the hyporheic zone. Water Resources Research, 53(1), 613-615. https://doi.org/10.1002/2016wr019488

Trauth, N., Musolff, A., Knöller, K., Kaden, U. S., Keller, T., Werban, U., \& Fleckenstein, J. H. (2018). River water infiltration enhances denitrification efficiency in riparian groundwater. Water Research, 130, 185-199. https://doi.org/10.1016/j.watres.2017.11.058

Uhlemann, S., Chambers, J., Wilkinson, P., Maurer, H., Merritt, A., Meldrum, P., et al. (2017). Four-dimensional imaging of moisture dynamics during landslide reactivation. Journal of Geophysical Research: Earth Surface, 122(1), 398-418. https://doi.org/10.1002/2016JF003983

Uhlemann, S., Hagedorn, S., Dashwood, B., Maurer, H., Gunn, D., Dijkstra, T., \& Chambers, J. (2016). Landslide characterization using $P$ and $S$ wave seismic refraction tomography-The importance of elastic moduli. Journal of Applied Geophysics, 134, 64-76. https://doi. org/10.1016/j.jappgeo.2016.08.014

Valois, R., Cousquer, Y., Schmutz, M., Pryet, A., Delbart, C., \& Dupuy, A. (2018). Characterizing stream-aquifer exchanges with self-potential measurements. Groundwater, 56(3), 437-450. https://doi.org/10.1111/gwat.12594

Vidon, P., \& Hill, A. R. (2006). A landscape-based approach to estimate riparian hydrological and nitrate removal functions. Journal of the American Water Resources Association, 42(4), 1099-1112. https://doi.org/10.1111/j.1752-1688.2006.tb04516.x

Vidon, P. G. F., \& Hill, A. R. (2004). Landscape controls on nitrate removal in stream riparian zones. Water Resources Research, 40(3), 1-14. https://doi.org/10.1029/2003WR002473

Wathelet, M. (2008). An improved neighborhood algorithm: Parameter conditions and dynamic scaling. Geophysical Research Letters, 35(9), L09301. https://doi.org/10.1029/2008GL033256

Wathelet, M., Jongmans, D., \& Ohrnberger, M. (2004). Surface-wave inversion using a direct search algorithm and its application to ambient vibration measurements. Near Surface Geophysics, 2(4), 211-221. https://doi.org/10.3997/1873-0604.2004018

Watlet, A., Kaufmann, O., Triantafyllou, A., Poulain, A., Chambers, J. E., Meldrum, P. I., et al. (2018). Imaging groundwater infiltration dynamics in the karst vadose zone with long-term ERT monitoring. Hydrology and Earth System Sciences, 22(2), 1563-1592. https://doi. org/10.5194/hess-22-1563-2018

Weatherill, J., Krause, S., Voyce, K., Drijfhout, F., Levy, A., \& Cassidy, N. (2014). Nested monitoring approaches to delineate groundwater trichloroethene discharge to a UK lowland stream at multiple spatial scales. Journal of Contaminant Hydrology, 158, 38-54. https://doi. org/10.1016/j.jconhyd.2013.12.001

Wildenschild, D., Jensen, K. H., Villholth, K., \& Illangasekare, T. H. (1994). A laboratory analysis of the effect of macropores on solute transport. Ground Water, 32(3), 381-389. https://doi.org/10.1111/j.1745-6584.1994.tb00655.x

Winter, T. C., Harvey, J. W., Lehn Franke, O., \& Alley, W. M. (1998). Groundwater and surface water: A single resource. U.S. Geological Survey. https://doi.org/10.3133/cir1139

Woessner, W. W. (2000). Stream and fluvial plain ground water interactions: Rescaling hydrogeologic thought. Ground Water, 38(3), 423429. https://doi.org/10.1111/j.1745-6584.2000.tb00228.x

Zarnetske, J. P., Haggerty, R., Wondzell, S. M., \& Baker, M. A. (2011). Dynamics of nitrate production and removal as a function of residence time in the hyporheic zone. Journal of Geophysical Research Biogeosciences, 116(G1), 1-12. https://doi.org/10.1029/2010JG001356

Zarnetske, J. P., Haggerty, R., Wondzell, S. M., Bokil, V. A., \& González-Pinzón, R. (2012). Coupled transport and reaction kinetics control the nitrate source-sink function of hyporheic zones. Water Resources Research, 48(11), 1-15. https://doi.org/10.1029/2012WR011894 